\title{
DENSITY OF PATHS OF ITERATED LÉVY TRANSFORMS OF BROWNIAN MOTION
}

\author{
MARC MALRIC ${ }^{1}$
}

\begin{abstract}
The Lévy transform of a Brownian motion $B$ is the Brownian motion $B^{(1)}$ given by $B_{t}^{(1)}=\int_{0}^{t} \operatorname{sgn}\left(B_{s}\right) \mathrm{d} B_{s}$; call $B^{(n)}$ the Brownian motion obtained from $B$ by iterating $n$ times this transformation. We establish that almost surely, the sequence of paths $\left(t \mapsto B_{t}^{(n)}\right)_{n \geqslant 0}$ is dense in Wiener space, for the topology of uniform convergence on compact time intervals.
\end{abstract}

Mathematics Subject Classification. 60g99, 60j65, 37a05, 37a50, 37a25.

Received February 24, 2009.

\section{INTRODUCTION}

Denote by $(\mathbb{W}, \mathcal{W}, \mu)$ the Wiener space: $\mathbb{W}$ is the space of all continuous functions $w$ on $[0, \infty[$ with $w(0)=0$; it is endowed with the topology of uniform convergence on compact sets; $\mathcal{W}$ is the Borel $\sigma$-field on $\mathbb{W}$, and $\mu$ is the Wiener measure.

A Brownian motion $B$ is a $(\mathbb{W}, \mathcal{W})$-valued random variable defined on some sample space $(\Omega, \mathcal{A}, \mathbb{P})$ and having law $\mathcal{L}(B)=\mathbb{P} \circ B^{-1}=\mu$. The Lévy transform of $B$ is the new Brownian motion $B_{t}^{\prime}=\int_{0}^{t} \operatorname{sgn} B_{s} \mathrm{~d} B_{s}$. Denote by $\mathbf{T}: \mathbb{W} \rightarrow \mathbb{W}$ the map such that $B^{\prime}=\mathbf{T} \circ B$; this map $\mathbf{T}$ is the Lévy transformation; it is defined up to $\mu$-negligibility (if $\mathbf{T}^{\prime}$ is another version, $\mathbf{T}^{\prime}=\mathbf{T}$ on a $\mu$-full set) and it preserves $\mu$ (i.e., $\mu \circ \mathbf{T}^{-1}=\mu$ ). These two properties, being well defined modulo $\mu$-null sets and invariance of $\mu$, allow the definition of the iterated Lévy transforms $\mathbf{T}^{n}: \mathbb{W} \rightarrow \mathbb{W}$ for all $n \geqslant 0$; and $\mathbf{T}^{n}$ also preserves $\mu$.

Understanding the asymptotic behavior of the dynamical system $(\mathbb{W}, \mathcal{W}, \mu, \mathbf{T})$ seems difficult; the question whether $\mathbf{T}$ is ergodic is still unsolved (see [1-3]). We shall establish that $\mathbf{T}$ is topologically recurrent: for $\mu$-almost all $w \in \mathbb{W}$, the orbit $\left\{\mathbf{T}^{k} w, k \geqslant 0\right\}$ is dense in $\mathbb{W}$. Topological recurrence is a necessary condition for ergodicity, but it is far from sufficient.

\section{Attainability}

The basic idea of our proof is expressed in the notion of attainability; the reason for this terminology will be explained later in this section. We state it in the context of the Lévy transformation, but everything in this section extends immediately to general dynamical systems (with a countable basis of open sets needed in Cor. 2.4).

\footnotetext{
Keywords and phrases. Brownian motion, Lévy transform, excursions, zeroes of Brownian motion, ergodicity.

1 15, avenue Gambetta, 94160 St-Mandé, France. marc.malric@gmail.com
} 
Definition 2.1. A measurable set $G \in \mathcal{W}$ is said to be attainable if for every $\eta>0$ there exist an integer $n \geqslant 0$ and a probability $\nu$ on $(\mathbb{W}, \mathcal{W})$ such that

(a1) $\nu$ is absolutely continuous w.r.t. $\mu$;

(a2) $\nu \circ\left(\mathbf{T}^{n}\right)^{-1}=\mu$;

(a3) $\nu(G)>1-\eta$.

Remark that (a2) is meaningful owing to (a1): if a measure $\nu$ on $\mathbb{W}$ is absolutely continuous w.r.t. $\mu$, then the image $\nu \circ\left(\mathbf{T}^{n}\right)^{-1}$ of $\nu$ by $\mathbf{T}^{n}$ makes sense, for it is insensitive to the choice of a version of $\mathbf{T}$. Remark also that if $G$ is attainable, every $G^{\prime} \in \mathcal{W}$ such that $G^{\prime} \supset G$ is attainable too.

Proposition 2.2. If $G \in \mathcal{W}$ is attainable, then for $\mu$-almost every $w \in \mathbb{W}$ the orbit $\left\{\mathbf{T}^{k} w, k \geqslant 0\right\}$ meets $G$.

Proof. The orbit of a path $w \in \mathbb{W}$ meets $G$ if and only if $w \in \bigcup_{k \geqslant 0} \mathbf{T}^{-k} G$; so, putting $F=\bigcup_{k \geqslant 0} \mathbf{T}^{-k} G$, we have to show that $\mu(F)=1$. It suffices to prove $\mu(F)>1-\eta$ for an arbitrary $\eta>0$. Given $\eta$, by attainability of $G$, we have an $n$ and a $\nu$ verifying (a1), (a2) and (a3). The definition of $F$ gives the inclusion $\mathbf{T}^{-n} F=\bigcup_{k \geqslant n} \mathbf{T}^{-k} G \subset F$; but these two sets $\mathbf{T}^{-n} F$ and $F$ have the same $\mu$-measure, because $\mathbf{T}$ preserves $\mu$. So their difference $N=F-\mathbf{T}^{-n} F$ is $\mu$-negligible, and also $\nu$-negligible by (a1). Writing $G \subset F=N \cup \mathbf{T}^{-n} F$ and taking $\nu$-measures yields $\nu(G) \leqslant \nu\left(\mathbf{T}^{-n} F\right)$; using (a3) and (a2) now gives $1-\eta<\mu(F)$.

Corollary 2.3. If every compact set $G \subset \mathbb{W}$ with $\mu(G)>0$ is attainable, $\mathbf{T}$ is ergodic.

Proof. By inner regularity of $\mu$, every non-negligible Borel set in $\mathbb{W}$ contains a non-negligible compact subset; so the hypothesis entails that all non-negligible Borel sets are attainable. Let $G$ be T-invariant with $\mu(G)>0$. Then $G$ is attainable, and Proposition 2.2 gives $\mu\left(\bigcup_{k \geqslant 0} \mathbf{T}^{-k} G\right)=1$, wherefrom $\mu(G)=1$ by $\mathbf{T}$-invariance of $G$. Thus $\mathbf{T}$ is ergodic.

Corollary 2.4. If every non-empty open subset of $\mathbb{W}$ is attainable, $\mu$-almost all $w \in \mathbb{W}$ have a dense orbit (i.e., $\mathbf{T}$ is topologically recurrent).

Proof. Apply Proposition 2.2 to all $G$ in a countable basis of open subsets of $\mathbb{W}$.

We have not been able to prove ergodicity of the Lévy transform via Corollary 2.3; the sequel is devoted to establishing that the Lévy transform satisfies the hypothesis of Corollary 2.4, and consequently is topologically recurrent.

We start by equivalently rephrasing the definition of an attainable set. Instead of introducing a probability $\nu$ on $\mathbb{W}$, we shall work with a $\mathbb{W}$-valued r.v. having law $\nu$. Such a r.v. is a stochastic process, started from 0 , with continuous paths. In this language, attainability is redefined as in the following proposition, whose proof is trivial:

Proposition 2.5. A set $G \in \mathcal{W}$ is attainable if and only if for each $\eta>0$ there exists, on some probability space $(\Omega, \mathcal{A}, \mathbb{P})$, a process $\Gamma$ such that

(A1) the law $\mathcal{L}(\Gamma)$ is absolutely continuous w.r.t. the Wiener measure $\mu$;

(A2) for some $n \geqslant 0$, the process $\mathbf{T}^{n} \circ \Gamma$ is a Brownian motion;

(A3) $\mathbb{P}[\Gamma \in G]>1-\eta$.

As was already the case with (a2) and (a1), remark that (A2) is meaningful owing to the absolute continuity (A1): if $\mathcal{L}(\Gamma) \ll \mu$, then $\mathbf{T} \circ \Gamma$ is well defined up to negligible events and $\mathcal{L}(\mathbf{T} \circ \Gamma) \ll \mu$. The former is proven by considering another version $\mathbf{T}^{\prime}$ and by observing that $\mathbb{P}\left[\mathbf{T}^{\prime} \circ \Gamma \neq \mathbf{T} \circ \Gamma\right]=\mathbb{P}\left[\Gamma \in\left\{\mathbf{T}^{\prime} \neq \mathbf{T}\right\}\right]=0$ because $\left\{\mathbf{T}^{\prime} \neq \mathbf{T}\right\}$ is $\mu$-negligible; the latter by $\mu$-invariance of $\mathbf{T}$. Inductively, all iterated Lévy transforms $\mathbf{T}^{n} \circ \Gamma$ are also a.s. well defined and have absolutely continuous laws. 
Definition 2.6. A stochastic process is tame if its law is absolutely continuous w.r.t. Wiener measure. (So a tame process is nothing but a $\mathbb{W}$-valued r.v. $U$ such that $\mathcal{L}(U) \ll \mu$ ).

If $V$ is tame, $\mathbf{T} \circ V$ is (a.s.) well defined, and is tame too. If $U$ and $V$ are two tame processes such that $\mathbf{T} \circ V=U$, we say that $V$ is a Lévy raise of $U$, or that $(U, V)$ is a Lévy raise.

A sequence of Lévy raises is a finite sequence $\left(U^{0}, U^{1}, \ldots, U^{n}\right)$ where $U^{0}$ is tame and each $U^{k+1}$ is a Lévy raise of $U^{k}$. Equivalently, $\left(U^{0}, U^{1}, \ldots, U^{n}\right)$ is a sequence of Lévy raises if and only if $U^{n}$ is tame and $U^{k}=\mathbf{T}^{n-k} U^{n}$ for each $k \in\{0, \ldots, n-1\}$.

Lévy raises will be more explicitly described in the next section; we first explain the meaning of attainability. According to Proposition 2.5, a set $G$ is attainable if there exists, on a suitable probability space, a Brownian motion $U^{0}$ (namely, $U^{0}=\mathbf{T}^{n} \circ \Gamma$ ) and a sequence $\left(U^{0}, \ldots, U^{n}\right)$ of Lévy raises (namely, $U^{k}=\mathbf{T}^{n-k} \circ \Gamma$ ) such that one has $\mathbb{P}\left[U^{n} \in G\right]>1-\eta$. In plain words, $G$ can be attained with arbitrarily high probability when one starts from a Brownian motion and performs finitely many Lévy raises; this is where the name attainable comes from.

To establish topological recurrence of $\mathbf{T}$ with Corollary 2.4, all we have to do is to attain each non-empty, open subset of $\mathbb{W}$ by a sequence of Lévy raises started from some Brownian motion. We shall first describe more precisely how some Lévy raises can be constructed.

\section{LÉVY RAISES}

Notation 3.1. Denote by $\mathbb{W}^{+}$(resp. $\mathbb{W}^{-}$) the subset of $\mathbb{W}$ consisting of all paths with values in $\mathbb{R}^{+}$(resp. $\mathbb{R}^{-}$).

The mapping $\mathbf{I}: \mathbb{W} \rightarrow \mathbb{W}^{-}$is the current minimum, defined by $(\mathbf{I} w)(t)=\min _{s \in[0, t]} w(s)$.

The mapping $\mathbf{J}: \mathbb{W} \rightarrow \mathbb{W}^{+}$is defined by $\mathbf{J} w=w-\mathbf{I} w$.

The next lemma recalls well-known properties of Brownian motion, essentially due to P. Lévy.

\section{Lemma 3.2.}

(a) For $\mu$-almost all $w \in \mathbb{W}$, one has $|w|=\mathbf{J T} w$;

(b) there exists a measurable functional $\mathbf{F}: \mathbb{W}^{+} \rightarrow \mathbb{W}$ such that, for $\mu$-almost all $w \in \mathbb{W}$, one has $\mathbf{F}|w|=\mathbf{T} w$ and $\mathbf{F} \mathbf{J} w=w$.

Proof.

(a) It suffices to verify that if $B$ is a Brownian motion, the processes $|B|$ and $\mathbf{J T} \circ B$ are indistinguishable. Tanaka's formula says that the Lévy transform $B^{\prime}$ of $B$ is given by $B^{\prime}=\mathbf{T} \circ B=|B|-L$, where $L$ denotes the local time of $B$ at the origin. For $t \geqslant 0$, put $g_{t}=\max \left\{s: s \in[0, t]\right.$ and $\left.B_{s}=0\right\}$. On the interval $[0, t]$, one has

$$
B^{\prime}=|B|-L \geqslant 0-L_{t}=\left|B_{g_{t}}\right|-L_{g_{t}}=B_{g_{t}}^{\prime},
$$

wherefrom $B_{g_{t}}^{\prime}=\inf _{[0, t]} B^{\prime}$. So $\left(\mathbf{I} \circ B^{\prime}\right)_{t}=B_{g_{t}}^{\prime}=-L_{t}$, that is, $\mathbf{I} \circ B^{\prime}=-L$, and $\mathbf{J T} \circ B=\mathbf{J} \circ B^{\prime}=B^{\prime}+L=|B|$. This proves (a);

(b) a possible choice for $\mathbf{F}$ is

$$
(\mathbf{F} w)(t)=w(t)-\lim _{\varepsilon \downarrow 0} \frac{1}{2 \varepsilon} \int_{0}^{t} \mathbf{1}_{\{w(s)<\varepsilon\}} \mathrm{d} s .
$$

The theory of Brownian local time (see for instance [3] p. 227) shows that $\mathbf{F}(|B|$ ) exists a.s. and $\mathbf{F}(|B|)=$ $|B|-L=\mathbf{T} \circ B$; so $\mathbf{F}|w|=\mathbf{T} w$ for a.a. $w$. Using (a), one has

$$
B^{\prime}=\mathbf{T} \circ B=\mathbf{F}(|B|)=\mathbf{F} \mathbf{J} \mathbf{T} \circ B=\mathbf{F J} \circ B^{\prime} ;
$$

since $B^{\prime}$ is a Brownian motion, $\mathbf{F} \mathbf{J} w=w$ for a.a. $w$ and $(\mathrm{b})$ is established. 
Corollary 3.3. Let $U$ and $V$ be two processes defined on the same probability space.

(a) If $V$ is tame, $|V|=\mathbf{J} \circ U$ a.s. on the event $\{\mathbf{T} \circ V=U\}$;

(b) if both $U$ and $V$ are tame, $\{\mathbf{T} \circ V=U\}=\{|V|=\mathbf{J} \circ U\}$ a.s.;

(c) $(U, V)$ is a Lévy raise if and only if $U$ and $V$ are tame and $|V|=\mathbf{J} \circ U$.

Proof.

(a) If $V$ is tame, Lemma 3.2 (a) gives $\mathbf{J T} \circ V=|V|$ a.s.; hence $|V|=\mathbf{J} \circ U$ on $\{\mathbf{T} \circ V=U\}$;

(b) if $U$ and $V$ are tame, Lemma 3.2 (b) entails $\mathbf{F J} \circ U=U$ a.s. and $\mathbf{T} \circ V=\mathbf{F}(|V|)$ a.s.; therefore, on $\{|V|=\mathbf{J} \circ U\}$, we have $\mathbf{T} \circ V=\mathbf{F}(|V|)=\mathbf{F} \mathbf{J} \circ U=U$ a.s. The reverse inclusion $\{\mathbf{T} \circ V=U\} \subset\{|V|=\mathbf{J} \circ U\}$ is given by (a);

(c) is a direct consequence of (b).

A Lévy raise starts from a tame process $U$ and produces a tame process $V$ such that $\mathbf{T} \circ V=U$. Corollary 3.3 (c) says that given $U$, any tame $V$ such that $|V|=\mathbf{J} \circ U$ a.s. is a Lévy raise of $U$. In other words, to perform a Lévy raise, $|V|$ must be taken equal to $\mathbf{J} \circ U$, but we are free to choose the signs of the excursions of $V$ away from zero, provided these choices yield a tame process $V$. Proposition 3.8 will show that if all but finitely many excursion signs are chosen at random by tossing a fair coin independently of $U$, then $(U, V)$ is a Lévy raise.

To handle excursion signs, we need to consider them as random variables; there is no canonical way to do that, so we arbitrarily choose the following definition.

Notation 3.4. For $w \in \mathbb{W}$ and $q>0$, denote by $Z(w)=\{s \geqslant 0: w(s)=0\}$ the set of zeros of $w$, and define $g_{q}(w)=\sup (Z(w) \cap[0, q]) \geqslant 0$ (last zero of $w$ before $q$ ) and $d_{q}(w)=\inf (Z(w) \cap[q, \infty[) \leqslant \infty$ (first zero of $w$ after $q)$.

Fix a dense sequence $\left(q_{n}\right)$ in the half-line $] 0, \infty\left[\right.$. To each $w \in \mathbb{W}$, we can attach the sequence $\left(e_{p}\right)$ of disjoint, open intervals, obtained from the sequence

$$
(] g_{q_{1}}, d_{q_{1}}[,] g_{q_{2}}, d_{q_{2}}[, \ldots,] g_{q_{n}}, d_{q_{n}}[, \ldots)
$$

by deleting an interval whenever it already occurs earlier in the sequence. The $e_{p}$ are the excursion intervals of $w$. There are $\mu$-a.s. infinitely many of them, and they are the connected components of the open set $[0, \infty[\backslash Z(w)$. The interval $e_{p}(w)$ will be called the $p$-th excursion interval of $w ; e_{p}$ is an interval-valued measurable map, defined on $(\mathbb{W}, \mathcal{W})$ up to $\mu$-negligibility.

Since $w$ does not vanish on $e_{p}(w)$, its sign remains constant on this interval. This sign will be denoted by $\mathbf{S}_{p}(w)$ and called the $p$-th excursion sign of $w$, and the sequence $\left(\mathbf{S}_{p}\right)$ will be called $\mathbf{S}$. So $\mathbf{S}$ is a measurable map from (a $\mu$-full subset of) $\mathbb{W}$ to $\{-1,+1\}^{\mathbb{N}}$. A path $w$ with infinitely many excursions is fully characterized by its absolute value $|w|$ and its excursion signs $\mathbf{S} w$. Encoding a path by its absolute value and its excursion signs is specially interesting for a Brownian motion $B$, since the random variables $|B|$ and $\mathbf{S} \circ B$ are independent. This is a consequence of excursion theory (see for instance Chap. XII of [3]), which also shows that the sequence $\left(\mathbf{S}_{p} \circ B\right)_{p \in \mathbb{N}}$ is i.i.d. and uniform on $\{-1,+1\}$. In other words, calling $\pi$ the probability on $\{-1,+1\}^{\mathbb{N}}$ which is the law of a fair coin-tossing, we have $\mathcal{L}(\mathbf{S} \circ B,|B|)=\pi \otimes \mathcal{L}(|B|)$.

When constructing a Lévy raise $V$ of a given tame process $U$, the signs $\mathbf{S} \circ V$ must be chosen so that $V$ is tame; the next two lemmas will enable us to check tameness of $V$ from the decomposition $(\mathbf{S} \circ V,|V|)$.

Lemma 3.5. Let $E$ and $F$ be two measurable spaces, and $\nu_{1}$ and $\nu_{2}$ two probabilities on $E$ such that $\nu_{1} \ll \nu_{2}$.

(a) For each measurable $f: E \rightarrow F$, the images $\nu_{1} \circ f^{-1}$ and $\nu_{2} \circ f^{-1}$ of $\nu_{1}$ and $\nu_{2}$ by $f$ satisfy $\nu_{1} \circ f^{-1} \ll$ $\nu_{2} \circ f^{-1}$;

(b) if $\rho$ is a probability on $F$, then $\rho \otimes \nu_{1} \ll \rho \otimes \nu_{2}$. 
Proof.

(a) If $A \subset F$ is negligible for $\nu_{2} \circ f^{-1}$, then $\nu_{2}\left(f^{-1} A\right)=0$, whence $\nu_{1}\left(f^{-1} A\right)=0$, and $A$ is negligible for $\nu_{1} \circ f^{-1}$.

(b) write $\nu_{1}=\phi \cdot \nu_{2}$ for some density $\phi$; then $\rho \otimes \nu_{1}=(1 \otimes \phi) \cdot\left(\rho \otimes \nu_{2}\right)$.

Lemma 3.6. Let $X$ be a tame process, and $Y$ a continuous process such that $|Y|=|X|$ and that $Y=X$ except for the signs of finitely many excursions. Then $Y$ is tame too.

Proof of Lemma 3.6. We will use the following notation: if $H$ is a subset of $\mathbb{N}$, put $H^{\mathrm{c}}=\mathbb{N} \backslash H$ and, for each sequence $S \in\{-1,+1\}^{\mathbb{N}}$, set $S^{H}=\left(S_{p}\right)_{p \in H}$ and $S^{H^{\mathrm{c}}}=\left(S_{p}\right)_{p \in H^{\mathrm{c}}}$. For $\sigma \in\{-1,+1\}^{H}, \tau \in\{-1,+1\}^{H^{\mathrm{c}}}$ and $u \in \mathbb{W}^{+}$, call $f_{H}(\sigma, \tau, u)$ the path $w \in \mathbb{W}$ such that $\mathbf{S}^{H}(w)=\sigma, \mathbf{S}^{H^{c}}(w)=\tau$ and $|w|=u$ (if $u$ has only finitely many excursions, ignore the superfluous information; anyway, this case will be negligible).

As a continuous process started from $0, Y$ is a measurable map from $(\Omega, \mathcal{A})$ to $(\mathbb{W}, \mathcal{W})$. Let $F \in \mathcal{W}$ be $\mu$-negligible; to prove the lemma it suffices to show $\mathbb{P}[Y \in F]=0$.

If $H$ is a finite subset of $\mathbb{N}$ and $B$ a Brownian motion, the triple $\left(\mathbf{S}^{H} \circ B, \mathbf{S}^{H^{c}} \circ B,|B|\right)$ is independent; hence

$$
\begin{aligned}
0 & =\mathbb{P}[B \in F]=\mathbb{P}\left[f_{H}\left(\mathbf{S}^{H} \circ B, \mathbf{S}^{H^{c}} \circ B,|B|\right) \in F\right] \\
& =\frac{1}{2^{|H|}} \sum_{\sigma \in\{-1,+1\}^{H}} \mathbb{P}\left[f_{H}\left(\sigma, \mathbf{S}^{H^{c}} \circ B,|B|\right) \in F\right] .
\end{aligned}
$$

As a consequence, $\mathbb{P}\left[f_{H}\left(\sigma, \mathbf{S}^{H^{c}} \circ B,|B|\right) \in F\right]=0$ for each finite subset $H$ of $\mathbb{N}$ and each $\sigma \in\{-1,+1\}^{H}$. As $X$ is tame, by Lemma 3.5 (a) one also has

$$
\mathbb{P}\left[f_{H}\left(\sigma, \mathbf{S}^{H^{c}} \circ X,|X|\right) \in F\right]=0 .
$$

Since $Y$ is obtained from $X$ by flipping the signs of finitely many excursions, there exists a r.v. $\mathcal{H}$, whose values are finite subsets of $\mathbb{N}$, such that $\mathbf{S}^{H^{c}} \circ Y=\mathbf{S}^{H^{c}} \circ X$ on the event $\{\mathcal{H}=H\}$. So the equality

$$
\mathbb{P}\left[\mathcal{H}=H, \mathbf{S}^{H} \circ Y=\sigma, f_{H}\left(\sigma, \mathbf{S}^{H^{c}} \circ X,|X|\right) \in F\right]=0,
$$

which stems trivially from $(*)$, can be rewritten as

$$
\mathbb{P}\left[\mathcal{H}=H, \mathbf{S}^{H} \circ Y=\sigma, f_{H}\left(\mathbf{S}^{H} \circ Y, \mathbf{S}^{H^{c}} \circ Y,|Y|\right) \in F\right]=0,
$$

that is, $\mathbb{P}\left[\mathcal{H}=H, \mathbf{S}^{H} \circ Y=\sigma, Y \in F\right]=0$. Summing first over all $\sigma \in\{-1,+1\}^{H}$ and then over all finite $H$ now yields $\mathbb{P}[Y \in F]=0$.

Corollary 3.7 (excursion-flipping principle). If $(U, V)$ is a Lévy raise, and if $V^{\prime}$ is a process obtained from $V$ by changing the signs of finitely many excursions of $V$, then $\left(U, V^{\prime}\right)$ is also a Lévy raise.

Proof. Immediate from Lemma 3.6 and Corollary 3.3 (c).

Proposition 3.8 (recipe for Lévy raises). Let $U$ be a tame process and $\tau$ a fair coin-tossing independent of $U$. If $V$ is any continuous process such that $|V|=\mathbf{J} \circ U$ and $\mathbf{S}_{p} \circ V=\tau_{p}$ except for $p$ in a (random) finite set of indices, then $V$ is tame and is a Lévy raise of $U$.

Proof. By Lemma 3.6 and Corollary 3.7, we may suppose that $\mathbf{S} \circ V=\tau$ holds identically.

By tameness of $U$, if $B$ is a Brownian motion, $\mathcal{L}(U) \ll \mathcal{L}(\mathbf{T} \circ B)$; Lemma 3.5 (a) with $f=\mathbf{J}$ and Lemma 3.2 (a) gives

$$
\mathcal{L}(|V|)=\mathcal{L}(\mathbf{J} \circ U) \ll \mathcal{L}(\mathbf{J} \mathbf{T} \circ B)=\mathcal{L}(|B|) .
$$


Using the independence of $\mathbf{S} \circ V=\tau$ and $|V|=\mathbf{J} \circ U$, one has by Lemma 3.5 (b)

$$
\mathcal{L}(\mathbf{S} \circ V,|V|)=\pi \otimes \mathcal{L}(|V|) \ll \pi \otimes \mathcal{L}(|B|)=\mathcal{L}(\mathbf{S} \circ B,|B|) ;
$$

using Lemma 3.5 (a) again with $f$ the map such that $f(\mathbf{S} \circ w,|w|)=w$, we obtain $\mathcal{L}(V) \ll \mathcal{L}(B)$, and $V$ is tame.

So both $U$ and $V$ are tame; as $|V|=\mathbf{J} \circ U$, Corollary 3.3 (c) asserts that $V$ is a Lévy raise of $U$.

In words, the recipe in Proposition 3.8 is: given $U$, put $|V|=\mathbf{J} \circ U$, and draw the signs of the excursions of $V$ according to a fair coin-tossing independent of $U$, except for a.s. finitely many excursions; those selected excursions can be assigned signs at will by any measurable procedure.

Our goal in the sequel is to establish topological recurrence by attaining all non-empty open subsets of $\mathbb{W}$ (Cor. 2.4): given an open $G$ and a Brownian motion $B$, to perform finitely many Lévy raises as in Proposition 3.8 so as to end up with a process which has property $G$ with high probability. Before getting down to work, we end this section with a small lemma, for later use.

Lemma 3.9. Given $t \geqslant 0$, let $U$ and $V$ be two independent tame processes; call $\Gamma$ the process equal to $U$ on $[0, t]$ and to $V-V_{t}+U_{t}$ on $[t, \infty[$.

(a) The process $\Gamma$ is tame too;

(b) if moreover $V$ is a Brownian motion, then, for each $n \geqslant 1$, on the interval $\left[t, \infty\left[\right.\right.$, the process $\left(\mathbf{T}^{n} \circ \Gamma\right)-$ $\left(\mathbf{T}^{n} \circ \Gamma\right)(t)$ is a Brownian motion independent of $U$.

Proof.

(a) Use Lemma 3.5: the law of $(U, V)$ is absolutely continuous w.r.t. $\mu \otimes \mu$, and $\Gamma=f(U, V)$ where, for $u$ and $v$ in $\mathbb{W}, f(u, v)$ is the concatenation of $u$ before $t$ with $v$ after $t$; so the law of $\Gamma$ is absolutely continuous w.r.t. the image of $\mu \otimes \mu$ by $f$, which equals $\mu$;

(b) if $\mathcal{F}$ denotes the filtration generated by $\Gamma$, define a larger filtration $\mathcal{G}$ by $\mathcal{G}_{s}=\mathcal{F}_{s}$ if $s<t$ and $\mathcal{G}_{s}=\mathcal{F}_{s} \vee \sigma(U)$ if $s \geqslant t$. In the enriched filtration $\mathcal{G}$, by independence, the process $\Gamma$ is still a semimartingale, and a Brownian motion after $t$; and $\mathbf{T}^{n} \circ \Gamma$ can be computed as a stochastic integral $\int H \mathrm{~d} \Gamma$, where $H=\prod_{i=0}^{n-1} \operatorname{sgn}\left(\mathbf{T}^{i} \circ \Gamma\right)$. Consequently, after $t, \mathbf{T}^{n} \circ \Gamma-\left(\mathbf{T}^{n} \circ \Gamma\right)(t)$ is a Brownian motion independent of $\mathcal{G}_{t}=\sigma(U)$.

\section{Partial LÉvy Raises}

We shall now deal with what we call partial Lévy raises; they are Lévy raises performed only on some (random) subset $[T, \infty[$ of the time axis, nothing being done on $[0, T]$ (preservation of the past).

Definition 4.1. Given two processes $U$ and $V$ and a random time $T$ with values in $[0, \infty]$, the triple $(U, V, T)$ will be called a partial Lévy raise if

- $U$ and $V$ are tame;

- on the event $\{0<T<\infty\}$, the random time $T$ is the end of an excursion of $U$ (that is, $U_{T}=0$ but, for some random $\varepsilon>0, U_{s} \neq 0$ for all $\left.s \in\right] T-\varepsilon, T[$ );

- for $s \in[0, T], V_{s}=U_{s}$ (preservation of the past);

- for $t \geqslant T,\left|V_{t}\right|=U_{t}-\min _{s \in[T, t]} U_{s}$.

By an abuse of language, we shall also say that a pair $(U, V)$ is a partial Lévy raise when there exists a random time $T$ such that $(U, V, T)$ is a partial Lévy raise. Notice that there is only one such $T$, namely, $T=\inf \left\{t: U_{t} \neq V_{t}\right\}$.

Observe that if $(U, V, T)$ is a partial Lévy raise, $U=\mathbf{T} \circ V$ on the event $\{T=0\}$, as a consequence of Corollary 3.3 (b). Also, trivially, $U=V$ on the event $\{T=\infty\}$. Remark also that $\mathbb{P}[T=t]=0$ for each deterministic $t>0$, since $T$ is the end of some excursion of $U$. 
If $\left(U^{i}, V^{i}\right)_{i \in I}$ is a countable family of Lévy raises and if $\left(E_{i}\right)_{i \in I}$ is a partition of $\Omega$ into countably many events, then, on setting $U=\sum_{i} U^{i} \mathbf{1}_{E_{i}}$ and $V=\sum_{i} V^{i} \mathbf{1}_{E_{i}}$, we obtain a new partial Lévy raise $(U, V)$. This fact stems trivially from the definition of partial Lévy raises; it will often be implicitly used in the sequel, to construct partial Lévy raises by a procedure which depends on the value of some discrete r.v.

Notation 4.2. Let $U$ be a process and $T$ a random time such that $T$ is the end of some excursion of $U$ on $\{0<T<\infty\}$. Denote by $U^{[T}$ and $\mathbf{J}^{[T} U$ the processes such that

$$
U_{t}^{[T}=\left\{\begin{array}{ll}
U_{T+t} & \text { if } T<\infty, \\
U_{t} & \text { if } T=\infty ;
\end{array} \quad\left(\mathbf{J}^{[T} U\right)_{t}= \begin{cases}0 & \text { if } t \leqslant T, \\
U_{t}-\inf _{s \in[T, t]} U_{s} & \text { if } t \geqslant T .\end{cases}\right.
$$

On the event $\{T<\infty\}$, one has $\left(\mathbf{J}^{[T} U\right)^{[T}=\mathbf{J} \circ U^{[T}$; but on $\{T=\infty\}, U^{[T}=U$ and $\mathbf{J}^{[T} U=0$.

Proposition 4.3 (recipe for partial Lévy raises). Let $U$ be a tame process and $T$ a random time which is the end of an excursion of $U$ on the event $\{0<T<\infty\}$.

(a) The process $U^{[T}$ is tame;

(b) let $V$ be a process such that

- $V=U$ on $[0, T]$;

- on $\left[T, \infty\left[\right.\right.$, one has $|V|=\mathbf{J}^{[T} U$ and, if $T<\infty$, the signs of the excursions of $V^{[T}$ (except possibly finitely many ones) are drawn according to a fair coin-tossing independent of $U$.

Then $V$ is tame, and $(U, V, T)$ is a partial Lévy raise.

Proof.

(a) Call $f_{t}: \mathbb{W} \rightarrow \mathbb{W}$ the map such that $f_{t}(w)(s)=w\left(d_{t}(w)+s\right)$, where $d_{t}(w)$ is the first sero of $w$ after $t$, and set $f_{\infty}(w)=w$. Since $U^{[T}=f_{t} \circ U$ on the event $\left\{T=d_{t}\right\}$, and since $\Omega=\bigcup_{t \in \mathbb{Q}^{+} \cup\{\infty\}}\left\{T=d_{t}\right\}$, it suffices to show that each $f_{t} \circ U$ is tame. This is given by Lemma 3.5, for $U$ is tame and $f_{t}$ preserves the Wiener measure;

(b) it suffices to check that $V$ is tame; $(U, V, T)$ will then satisfy the definition of a Lévy raise. By Lemma 3.6, we may change the signs of finitely many excursions of $V$; this allows us to suppose that the signs of all excursions of $V$ after $T$ are given by some coin-tossing $\tau$ independent of $U$. On the event $\left\{T=d_{t}\right\}$, one can write $V=h_{t}(U, \tau)$ where the functional $h_{t}$ is such that $h_{t}(B, \tau)$ is a Brownian motion if $B$ is a Brownian motion independent of $\tau$. Since $U$ and $\tau$ are independent and $U$ is tame, Lemma 3.5 says that $h_{t}(U, \tau)$ is tame. Tameness of $V$ then follows by the same argument as in (a).

Corollary 4.4. Let $U$ be a tame process and $S \leqslant \infty$ a random time such that $S$ is the end of an excursion of $U$ on the event $\{0<S<\infty\}$. Let $V$ be a process such that

- $V=U$ on $[0, S]$;

- on the event $\{S<\infty\}, V^{[S}$ is a partial Lévy raise of $U^{[S}$, prepared according to the recipe from Proposition 4.3 (b) (with some random time $T$ which is the end of some excursion of $U^{[S}$ if $0<T<\infty$, and with all but finitely many signs drawn independently of $U$ ).

Then $(U, V, S+T)$ is a partial Lévy raise.

Proof. By construction, $V=U$ on $[0, S+T]$, and on $[S+T, \infty[$ the signs of the excursions of $V$ are chosen according to the recipe from Proposition 4.3 because they are the signs of the excursions of $V^{[S}$ on $[T, \infty[$.

Propositions 3.8 and 4.3 will be used to construct new processes in situations where other processes are already given. An independent coin-tossing is needed; this extra randomness may not be available in the original sample space $(\Omega, \mathcal{A}, \mathbb{P})$, which may have to be "enlarged", that is, replaced with another $(\bar{\Omega}, \overline{\mathcal{A}}, \overline{\mathbb{P}})$ such that $\overline{\mathcal{A}}$ contains both an isomorphic copy of $\mathcal{A}$ and an independent coin-tossing. This is innocuous, because in the end, we are only interested in existence in law of the set of processes we are dealing with. But rigorously speaking, Proposition 4.5 and similar ones should be stated as: "on a suitable extension of the sample space, there exists a sequence of Lévy raises etc.". 
Recall from the end of Section 2 that an attainable set can be reached with high probability by a finite sequence of Lévy raises started from a Brownian motion. It will be convenient to replace Lévy raises with partial Lévy raises, and to do so with as slight a perturbation as possible. This is achieved by the next proposition.

Proposition 4.5. Suppose given a real number $t>0$, a r.v. $\varepsilon>0$, and a finite sequence of partial Lévy raises, that is, a sequence $\left(X^{0}, X^{1}, \ldots, X^{n}\right)$ such that, for each $i \in\{1, \ldots, n\}$, the pair $\left(X^{i-1}, X^{i}\right)$ is a partial Lévy raise.

There exists a sequence $\left(Y^{0}, \ldots, Y^{n}\right)$ of Lévy raises such that, for all $s \in[0, t],\left|Y_{s}^{n}-X_{s}^{n}\right|<\varepsilon$ and $\left|Y_{s}^{0}\right|=$ $\left|X_{s}^{0}\right|$.

All this section is devoted to proving Proposition 4.5. The instant $t>0$ is fixed in the rest of the section, and we are only interested in what happens on the interval $[0, t]$. For instance, the notion of an $\alpha$-double defined below depends on $t$; it could be called a $(t, \alpha)$-double, but it is understood that $t$ is implied everywhere.

In the next definition, if $e$ is an excursion of a path, with excursion interval $] a, b[$, we put $g(e)=a$ (debut of the excursion) and $d(e)=b$ (end of the excursion).

Definition 4.6. For $u$ and $v$ in $\mathbb{W}$ and $\alpha>0$, we say that $v$ is an $\alpha$-double of $u$ if

$\left(1_{\alpha}\right)$ on the interval $[0, t]$, one has $|v-u|<\alpha$;

$\left(2_{\alpha}\right)$ for every excursion $e$ of $u$ such that $|e(s)|>2 \alpha$ for some $s \in[0, t]$, there exists an excursion $e^{\prime}$ of $v$ whose interval contains the set $\{s: s \leqslant t$ and $|e(s)|>\alpha\}$ and such that $\left|g\left(e^{\prime}\right)-g(e)\right|<\alpha$ and $\left|d\left(e^{\prime}\right) \wedge t-d(e) \wedge t\right|<\alpha$.

Condition $\left(2_{\alpha}\right)$ is rather technical; it will be used only later, in the proof of Proposition 4.8. Remark that in $\left(2_{\alpha}\right)$ the excursion $e$ may be positive or negative, that $e^{\prime}$ is unique, has the same sign as $e$ and its amplitude must be $>2 \alpha$, and that the set $\{s: s \leqslant t$ and $|e(s)|>\alpha\}$ is not necessary connected.

If $v$ is an $\alpha$-double of $u$, it is also a $\beta$-double of $u$ for all $\beta>\alpha$; and any $v^{\prime}$ equal to $v$ on $[0, t]$ is an $\alpha$-double of any $u^{\prime}$ equal to $u$ on $[0, t]$. But notice that $\left(2_{\alpha}\right)$ it is not symmetric: $u$ and $v$ do not play similar roles; if $v$ is an $\alpha$-double of $u, u$ need not be an $\alpha$-double of $v$.

Definition 4.7. Given two tame processes $U$ and $V$ and a r.v. $\alpha>0$, we say that $V$ is an $\alpha$-double of $U$ if the path $V(\omega)$ is an $\alpha(\omega)$-double of $U(\omega)$ for a.a. $\omega \in \Omega$. If this only holds for a.a. $\omega$ in some event $E$, we say that $V$ is an $\alpha$-double of $U$ on $E$.

Proposition 4.5 will be proven by repeatedly applying the same argument, namely, the approximation property given by the first part of Proposition 4.8.

Proposition 4.8. Given a partial Lévy raise $(U, V)$ and a r.v. $\varepsilon>0$, there exists a r.v. $\delta>0$ with the following property: for every $\delta$-double $U^{\prime}$ of $U$, there exists a Lévy raise $\left(\tilde{U}, V^{\prime}\right)$ such that $|\tilde{U}|=\left|U^{\prime}\right|$ on $[0, t]$ and that $V^{\prime}$ is an $\varepsilon$-double of $V$.

Moreover, if $(U, V)$ is a Lévy raise, one can always take $\tilde{U}=U^{\prime}: \delta$ can be chosen so that for every $\delta$-double $U^{\prime}$ of $U$, there exists an $\varepsilon$-double $V^{\prime}$ of $V$ such that $\left(U^{\prime}, V^{\prime}\right)$ is a Lévy raise.

Proof of Proposition 4.5 (Prop. 4.8 is admitted). In the framework of Proposition 4.5, we are given a finite sequence $\left(X^{0}, \ldots, X^{n}\right)$ of partial Lévy raises and a r.v. $\varepsilon>0$. First, by backward induction, construct a sequence $\left(\varepsilon_{0}, \ldots, \varepsilon_{n}\right)$ as follows: set $\varepsilon_{n}=\varepsilon$, and, after $\varepsilon_{i}$ has been defined, define $\varepsilon_{i-1}$ to be a $\delta$ given by Proposition 4.8 applied to $(U, V)=\left(X^{i-1}, X^{i}\right)$ and $\varepsilon=\varepsilon_{i}$.

Then, define by forward induction two sequences $\left(Z^{0}, \ldots, Z^{n}\right)$ and $\left(R^{1}, \ldots, R^{n}\right)$ according to the following procedure. First, $\left(Z^{0}, R^{1}\right)$ is a Lévy raise $\left(\tilde{U}, V^{\prime}\right)$ given by Proposition 4.8 applied to $(U, V)=\left(X^{0}, X^{1}\right), \varepsilon=\varepsilon_{1}$, $\delta=\varepsilon_{0}$ and $U^{\prime}=X_{0}$. Then, after $\left(Z^{i-1}, R^{i}\right)$ has been defined, define $\left(Z^{i}, R^{i+1}\right)$ as a Lévy raise $\left(\tilde{U}, V^{\prime}\right)$ produced by Proposition 4.8 with $(U, V)=\left(X^{i}, X^{i+1}\right), \varepsilon=\varepsilon_{i+1}, \delta=\varepsilon_{i}$ and $U^{\prime}=R^{i}$; this is possible because $R^{i}$ is an $\varepsilon_{i}$-double of $X^{i}$ owing to the preceding step. Last, after $\left(Z^{n-1}, R^{n}\right)$ has been defined, put $Z^{n}=R^{n}$. For 
$i \in\{1, \ldots, n\}$, the following properties hold by construction: $\left(Z^{i-1}, R^{i}\right)$ is a Lévy raise, $\left|Z^{0}\right|=\left|X^{0}\right|$ on $[0, t]$, $\left|Z^{i}\right|=\left|R^{i}\right|$ on $[0, t]$, and $R^{i}$ is an $\varepsilon_{i}$-double of $X^{i}$.

For $1 \leqslant i \leqslant n, Z^{i}$ and $R^{i}$ are tame and have the same absolute value on $[0, t]$; therefore, $\mathbf{T} \circ Z^{i}=\mathbf{T} \circ R^{i}$ on $[0, t]$; now $\mathbf{T} \circ R^{i}=Z^{i-1}$, so $\mathbf{T} \circ Z^{i}=Z^{i-1}$ on $[0, t]$. By induction, this implies $\mathbf{T}^{n-i} \circ Z^{n}=Z^{i}$ on $[0, t]$ for $0 \leqslant i \leqslant n$.

Consequently, the finite sequence $\left(Y^{0}, \ldots, Y^{n}\right)$ of Lévy raises defined by $Y^{i}=\mathbf{T}^{n-i} \circ Z^{n}$ satisfies $Y^{i}=Z^{i}$ on $[0, t]$ and meets the requirement of Proposition 4.5: since $Y^{n}=R^{n}$ is an $\varepsilon_{n}$-double of $X^{n}$, one has $\left|Y^{n}-X^{n}\right|<\varepsilon$ on $[0, t]$ by $\left(1_{\alpha}\right)$ in the definition of an $\varepsilon$-double; and since $Y^{0}=Z^{0}$ on $[0, t],\left|Y^{0}\right|=\left|X^{0}\right|$ on that interval.

Notice that the preceding proof involves only the first part of Proposition 4.8 (the second part is set aside for later use) and only condition $\left(1_{\alpha}\right)$ in the definition of a double. Condition $\left(2_{\alpha}\right)$ (which admittedly is rather unpleasant) will be used when proving Proposition 4.8, which would be false if the definition of a double were replaced by the sole uniform approximation condition $\left(1_{\alpha}\right)$.

Notice also that the analogue of Proposition 4.8 with $\varepsilon=0$ and $\delta=0$ is false: if $(U, V)$ is a Lévy raise and $U^{\prime}$ a tame process such that $U^{\prime}=U$ on $[0, t]$, it may happen that there exists no tame process $V^{\prime}$ whatsoever such that $V^{\prime}=V$ on $[0, t]$ and $\mathbf{T} \circ V^{\prime}=U^{\prime}$. For a counter-example, consider the case that the whole process $\left(U_{t+s}^{\prime}-U_{t}^{\prime}\right)_{s \geqslant 0}$ is a functional $f$ of the signs of the excursions of $V$ which terminate before $t$. No tame process $V^{\prime}$ equal to $V$ on $[0, t]$ can satisfy $\mathbf{T} \circ V^{\prime}=U^{\prime}$ because, if $B$ is a Brownian motion, the event that the increments of $\mathbf{T} \circ B$ after $t$ are $f$ of the signs of the excursions of $B$ before $t$ is a negligible event.

The rest of this section is devoted to proving Proposition 4.8 (and consequently Prop. 4.5 too). One of our tools will be the next lemma.

Lemma 4.9. Let $V$ be a tame process. For each $r>0$, there exists a r.v. $\alpha>0$ such that, calling $] g, d[$ the interval of the excursion of $V$ straddling $r$, one has $2 \alpha<d-g$ and

$$
\forall s \in] g, d\left[\quad\left|V_{s}\right|>(s-g) \wedge(d-s) \wedge \alpha .\right.
$$

Proof of Lemma 4.9. As the property to be established is almost sure, we may suppose that $V$ is a Brownian motion. A well-known property of Brownian excursions (see for instance Chap. XII, Sect. 4 of [3]) asserts that near its debut $g$, the excursion leaves 0 as does a 3-Bessel; in particular, this implies existence of some r.v. $\alpha_{1}>0$ such that $\left|V_{s}\right|>s-g$ for all $\left.s \in\right] g, g+\alpha_{1}$ [; we may further require $2 \alpha_{1}<d-g$. Similarly, by temporal symmetry of Itô's measure of excursions, $\left|V_{s}\right|>d-s$ for all $\left.s \in\right] d-\alpha_{2}, d\left[\right.$, where $\alpha_{2}$ is some r.v. such that $0<2 \alpha_{2}<d-g$. Last, on the compact, non-empty interval $\left[g+\alpha_{1}, d-\alpha_{2}\right]$, one has $|V|>\alpha_{3}>0$, because $V$ is continuous and does not vanish. It now suffices to put $\alpha=\alpha_{1} \wedge \alpha_{2} \wedge \alpha_{3}$ and the lemma follows.

Proof of Proposition 4.8. We are given a partial Lévy raise $(U, V, T)$ and a r.v. $\varepsilon>0$. Define a process $I$ by $I_{s}=0$ if $s \leqslant T$ and $I_{s}=\inf _{r \in[T, s]} U_{r}$ if $s \geqslant T$; observe that $U-I \geqslant 0$ after $T$, and only finitely many excursions of $U-I$ starting in the finite interval $] T \wedge t, t]$ can reach the value $\varepsilon / 4$. Call $\ell_{1}, \ldots, \ell_{p}$ these excursions (in chronological order; the r.v. $p$ is set to 0 when there are no such excursions). The process $I$ is constant during each excursion interval $] g\left(\ell_{j}\right), d\left(\ell_{j}\right)\left[\left(\right.\right.$ call $I\left(\ell_{j}\right)$ this constant value), the sequence $\left(0, I\left(\ell_{1}\right), \ldots, I\left(\ell_{p}\right)\right)$ is strictly decreasing, and for $\alpha>0$ the inequalities $I\left(g\left(\ell_{j}\right)-\alpha\right)>I\left(\ell_{j}\right)$ and $I\left(d\left(\ell_{j}\right)+\alpha\right)<I\left(\ell_{j}\right)$ are strict. By definition of a partial Lévy raise, each $\ell_{j}$ is also the absolute value of an excursion of $V$, to which Lemma 4.9 applies. Last, observe that $d_{t}(V)$, the first zero of $V$ after $t$, is a.s. finite.

These remarks make it possible to choose some r.v. $\delta>0$ satisfying the following six requirements:

$\left(1_{\delta}\right) 10 \delta<\varepsilon$

$\left(2_{\delta}\right)$ for all $r$ and $s$ in $\left[0, d_{t}(V)\right],|r-s|<2 \delta \Rightarrow\left|V_{r}-V_{s}\right|<\varepsilon / 4$;

$\left(3_{\delta}\right)$ for each $j \in\{1, \ldots, p\}, 4 \delta<I\left(\ell_{j-1}\right)-I\left(\ell_{j}\right)$ (with the convention $I\left(\ell_{0}\right)=0$ );

$\left(4_{\delta}\right)$ for each $j \in\{1, \ldots, p\}, 2 \delta<I\left(g\left(\ell_{j}\right)-\varepsilon\right)-I\left(\ell_{j}\right)$; 
$\left(5_{\delta}\right)$ for each $j \in\{1, \ldots, p\}$ such that $d\left(\ell_{j}\right)<t, 2 \delta<I\left(\ell_{j}\right)-I\left(\left(d\left(\ell_{j}\right)+\varepsilon\right) \wedge t\right)$;

$\left(6_{\delta}\right)$ for each $j \in\{1, \ldots, p\}, 4 \delta<d\left(\ell_{j}\right)-g\left(\ell_{j}\right)$ and for all $\left.s \in\right] g\left(\ell_{j}\right), d\left(\ell_{j}\right)[$,

$$
U_{s}-I_{s}=\left|V_{s}\right|>s-g\left(\ell_{j}\right) \wedge d\left(\ell_{j}\right)-s \wedge 2 \delta .
$$

(On the event $\{p=0\}$, only $\left(1_{\delta}\right)$ and $\left(2_{\delta}\right)$ are demanded; the other ones are vacuously satisfied).

With such a $\delta$, we are going to show that the conclusion of Proposition 4.8 holds. So, from now on, not only are $t, U, V, T$ and $\varepsilon$ fixed, but so are also $\delta$, which satisfies the six conditions, and $U^{\prime}$, a given $\delta$-double of $U$.

Call $e_{1}, \ldots, e_{k}$ (in chronological order) the excursions of $U$ with amplitude $>2 \delta$ which start before $t \wedge T$; call also $e_{1}^{\prime}, \ldots, e_{k}^{\prime}$ the excursions of $U^{\prime}$ which correspond to the $e_{i}$ by $\left(2_{\alpha}\right)$. Choose (measurably) $2 k$ excursions $f_{1}, h_{1}, \ldots, f_{k}, h_{k}$ of $\left|U^{\prime}\right|$, in chronological order, so that

- $] g\left(f_{i}\right), d\left(f_{i}\right)[\subset] g\left(e_{i}^{\prime}\right)-\delta, g\left(e_{i}^{\prime}\right)[$ and $] g\left(h_{i}\right), d\left(h_{i}\right)[\subset] d\left(e_{i}^{\prime}\right), d\left(e_{i}^{\prime}\right)+\delta[;$

- the amplitudes of these $2 k$ excursions are chronologically increasing (the amplitude of $f_{1}$ is less that that of $h_{1}$, which is in turn less than that of $f_{2}$, etc.) and all these $2 k$ amplitudes are less than $\delta$.

This is possible because $U^{\prime}$ is tame (definition of a $\delta$-double), and because each Brownian excursion is immediately preceded and followed by infinitely many arbitrarily small other excursions.

Call $T^{\prime}$ the end of the excursion $h_{k}$ (put $T^{\prime}=0$ on the event $\{k=0\}$, and observe that $T^{\prime}$ is finite).

Set $\tilde{U}=U^{\prime}$, except for the signs of some excursions:

- $f_{1}, h_{1}, \ldots, f_{k}, h_{k}$ are made $<0$;

- all other excursions before $T^{\prime}$ and taller than $f_{1}$ (this includes $e_{1}^{\prime}, \ldots, e_{k}^{\prime}$ ) are made $>0$.

As $\tilde{U}$ differs from $U^{\prime}$ in the signs of finitely many, measurably chosen, excursions, $\tilde{U}$ is tame by Lemma 3.6. Remark also that $\tilde{U}>-3 \delta$ on the interval $[0, T \wedge t]$. Indeed, every $s \leqslant T \wedge t$ such that $\left|U_{s}\right|>2 \delta$ is in one of the $e_{i}$, and also, by $\left(2_{\alpha}\right)$, in the corresponding excursion $e_{i}^{\prime}$; so $\tilde{U}_{s}>0$. And for $s \leqslant T \wedge t$ such that $\left|U_{s}\right| \leqslant 2 \delta$, using $\left(1_{\alpha}\right)$ one has $\left|\tilde{U}_{s}\right|=\left|U_{s}^{\prime}\right|<\left|U_{s}\right|+\delta<3 \delta$.

Observe that when $T=0, k$ and $T^{\prime}$ are null too, and $\tilde{U}$ is nothing but $U^{\prime}$; consequently, proving the first sentence of Proposition 4.8 with this $U^{\prime}$ suffices to automatically entail the second sentence.

Define as follows a process $V^{\prime}$. First, its absolute value is $\left|V^{\prime}\right|=\mathbf{J} \circ \tilde{U}$. Then, the signs $\mathbf{S} \circ V^{\prime}$ of the excursions of $V^{\prime}$ are drawn at random according to the uniform law $\pi$, independently of $\tilde{U}$. Last, the signs of finitely many excursions of $V^{\prime}$ are redefined as specified below, thus overriding the random assignment previously made. According to Proposition 3.8, this process $V^{\prime}$ will be a Lévy raise of $\tilde{U}$.

There are $k+p$ excursions of $V^{\prime}$ whose signs will be prescribed according to some particular procedure.

The first $k$ of them correspond to $e_{1}, \ldots, e_{k}$ (and to $e_{1}^{\prime}, \ldots, e_{k}^{\prime}$ ) in the following manner. As a consequence of the choice of the signs of the excursions of $\tilde{U}$ before $T^{\prime}$, for each $i \in\{1, \ldots, k\}$ the process $\mathbf{I} \circ \tilde{U}$ is constant on some interval $\left[a_{i}, b_{i}\right]$, where $a_{i}$ is the time when $f_{i}$ is extremal and where $b_{i}=\inf \left\{s>a_{i}: \tilde{U}_{s}=\tilde{U}_{a_{i}}\right\}$ occurs during $h_{i}$. So $\mathbf{J} \circ \tilde{U}$ and also $V^{\prime}$ have an excursion $e_{i}^{\prime \prime}$ starting at time $a_{i}$ and ending at $b_{i}$. This excursion of $V^{\prime}$ is assigned the same sign as the excursion $e_{i}$ of $U$ (which is also an excursion of $V$, for $e_{i}$ occurs before $T$ and $(U, V, T)$ is a Lévy raise).

The other $p$ excursions of $V^{\prime}$ with prescribed signs correspond to the excursions $\ell_{j}$ introduced at the beginning of this proof. For $j \in\{1, \ldots, p\}$, the excursion $\ell_{j}$ of $U-I$ starts at $g\left(\ell_{j}\right)$ and ends at $d\left(\ell_{j}\right)$; on this interval, the value $I\left(\ell_{j}\right)$ of $I$ remains constant, and less that $-4 \delta$ by $\left(3_{\delta}\right)$. Owing to $\left(4_{\delta}\right)$, at time $T \vee g\left(\ell_{j}\right)-\varepsilon, U$ is above the value $I\left(\ell_{j}\right)+2 \delta$, and by $\left(6_{\delta}\right)$, at time $g\left(\ell_{j}\right)+2 \delta, U$ is again above the value $I\left(\ell_{j}\right)+2 \delta$. So $\tilde{U}$, which is minorated by $-3 \delta$ before $T \wedge t$ and $\delta$-close to $U$ on $[T \wedge t, t]$, also has a current minimum between $T \vee\left(g\left(\ell_{j}\right)-\varepsilon\right)$ and $g\left(\ell_{j}\right)+2 \delta$, and this minimal value $m_{j}$ is $\delta$-close to $U\left(g\left(\ell_{j}\right)\right)=I\left(\ell_{j}\right)$. Then, by $\left(6_{\delta}\right)$ again, on the interval $\left[g\left(\ell_{j}\right)+2 \delta, d\left(\ell_{j}\right)-2 \delta\right], U$ remains above $I\left(\ell_{j}\right)+2 \delta$, and a fortiori above $m_{j}+\delta$; consequently $\tilde{U}>m_{j}$ on $\left[g\left(\ell_{j}\right)+2 \delta, d\left(\ell_{j}\right)-2 \delta\right] \cap[0, t]$. Last, if $d\left(\ell_{j}\right)<t$, at time $\left(d\left(\ell_{j}\right)+\varepsilon\right) \wedge t\left(5_{\delta}\right)$ says that $I$ is less than $I\left(\ell_{j}\right)-2 \delta$, and a fortiori less than $m_{j}-\delta$; hence, at that time, $\mathbf{I} \circ \tilde{U}<m_{j}$ if $d\left(\ell_{j}\right)<t$. All in all, this shows that $\tilde{U}-\mathbf{I} \circ \tilde{U}$ has an excursion which starts between $g\left(\ell_{j}\right)-\varepsilon$ and $g\left(\ell_{j}\right)+2 \delta$, ends after $d\left(\ell_{j}\right)-2 \delta$ and, if $d\left(\ell_{j}\right)<t$, 
before $d\left(\ell_{j}\right)+\varepsilon$, and resembles $\ell_{j}$ on the interval $\left[g\left(\ell_{j}\right)+2 \delta, d\left(\ell_{j}\right)-2 \delta\right] \cap[0, t]$. Call $\ell_{j}^{\prime}$ this excursion of $\mathbf{J} \circ \tilde{U}$. Since $\left|m_{j}-I\left(\ell_{j}\right)\right|<\delta$ and $I\left(\ell_{j-1}\right)-I\left(\ell_{j}\right)>4 \delta$ by $\left(3_{\delta}\right)$, one has $m_{j} \neq m_{j-1}$ for $j \geqslant 2$; as $m_{j}$ is the value of $\mathbf{I} \circ \tilde{U}$ during $\ell_{j}^{\prime}$, the excursion $\ell_{j}^{\prime}$ cannot be the same as the excursion $\ell_{j-1}^{\prime}$. So we may - and do - assign to the excursion $\ell_{j}^{\prime}$ of $V^{\prime}$ the same sign as the excursion $\ell_{j}$ of $V$.

According to Proposition 3.8, $\left(\tilde{U}, V^{\prime}\right)$ is a Lévy raise. There remains to show that $V^{\prime}$ is an $\varepsilon$-double of $V$.

First, to check condition $\left(2_{\alpha}\right)$, we have to consider all excursions of $V$ reaching the value $\pm 2 \varepsilon$ before time $t$. Any such excursion occurring before $T$ is also an excursion of $U$, because $U=V$ on $[0, T]$ since $(U, V, T)$ is a partial Lévy raise. Now, using $\left(1_{\delta}\right)$, any excursion of $U$ occurring before $T$ and reaching amplitude $2 \varepsilon$ before $t$ is one of the $e_{i}$; as explained above, $V^{\prime}$ has a corresponding excursion $e_{i}^{\prime \prime}$, with the same sign as the excursion $e_{i}$ of $U$ and $V$, starting at a time $a_{i}$ which belongs to $f_{i}$ and ending at $b_{i}$ which belongs to $h_{i}$. Owing to the choice of $f_{i}$ and $h_{i}$, one has $\left|a_{i}-g\left(e_{i}^{\prime}\right)\right|<\delta$ and $\left|b_{i}-d\left(e_{i}^{\prime}\right)\right|<\delta$. As $U^{\prime}$ is a $\delta$-double of $U$, condition $\left(2_{\alpha}\right)$ gives $\left|g\left(e_{i}^{\prime}\right)-g\left(e_{i}\right)\right|<\delta$ and $\left|d\left(e_{i}^{\prime}\right) \wedge t-d\left(e_{i}\right) \wedge t\right|<\delta$; so finally $\left|g\left(e_{i}^{\prime \prime}\right)-g\left(e_{i}\right)\right|<2 \delta<\varepsilon$ and $\left|d\left(e_{i}^{\prime \prime}\right) \wedge t-d\left(e_{i}\right) \wedge t\right|<2 \delta<\varepsilon$. Next, we verify that any time $s \leqslant t$ belonging to $e_{i}$ and such that $\left|V_{s}\right|>2 \varepsilon$, also belongs to $e_{i}^{\prime \prime}$. Such an $s$ belongs to $e_{i}^{\prime}$ by definition of $e_{i}^{\prime}$ and because $\left|U_{s}\right|=\left|V_{s}\right|>2 \varepsilon>2 \delta$; now, by the choice of $f_{i}$ and $h_{i}$, the interval ] $a_{i}, b_{i}[$ contains ]$g\left(e_{i}^{\prime}\right), d\left(e_{i}^{\prime}\right)\left[\right.$, so $s$ also belongs to $e_{i}^{\prime \prime}$. This establishes $\left(2_{\alpha}\right)$ for the excursions of $V$ before $T$.

We now prove $\left(2_{\alpha}\right)$ for the excursions of $V$ after $T$. Any excursion of $V$ posterior to $T$ and reaching the value $\pm 2 \varepsilon$ before $t$ must be one of the $\ell_{j}$ used when defining $\delta$. We have seen that the corresponding excursion $\ell_{j}^{\prime}$ of $V^{\prime}$ satisfies $\left|g\left(\ell_{j}^{\prime}\right)-g\left(\ell_{j}\right)\right|<\varepsilon$ and $\left|d\left(\ell_{j}^{\prime}\right) \wedge t-d\left(\ell_{j}\right) \wedge t\right|<\varepsilon$. If an $s \leqslant t$ belongs to this $\ell_{j}$ and is such that $\left|V_{s}\right|>\varepsilon$, then $s$ also belongs to the smaller interval $\left[g\left(\ell_{j}\right)+2 \delta, d\left(\ell_{j}\right)-2 \delta\right]$ because $\left(2_{\delta}\right)$ forbids $s$ to be close to the zeros $g\left(\ell_{j}\right)$ and $d\left(\ell_{j}\right)$ of $V$. As $\left[g\left(\ell_{j}\right)+2 \delta, d\left(\ell_{j}\right)-2 \delta\right] \cap[0, t]$ is included in the excursion $\ell_{j}^{\prime}, s$ belongs to $\ell_{j}^{\prime}$ and $\left(2_{\alpha}\right)$ is fully established.

The only point which remains to be proven is $\left(1_{\alpha}\right)$, namely, $\left|V^{\prime}-V\right|<\varepsilon$ on $[0, t]$. Here again, we separately consider the intervals $[0, T \wedge t]$ and $] T \wedge t, t]$.

First, on $[0, T \wedge t]$, we know that $\tilde{U}>-3 \delta$, wherefrom $\mathbf{I} \circ \tilde{U}>-3 \delta$ and

$$
|| U^{\prime}|-| V^{\prime}||=|| \tilde{U}|-| \mathbf{J} \circ \tilde{U}|| \leqslant|\tilde{U}-\mathbf{J} \circ \tilde{U}|=|\mathbf{I} \circ \tilde{U}|<3 \delta .
$$

Using now $V=U$ on $[0, T]$ and $\left|U^{\prime}-U\right|<\delta$ on $[0, t]$, we get ||$V^{\prime}|-| V||<4 \delta$.

We now consider two sub-cases. For an $s \in[0, T \wedge t]$ such that $\left|V_{s}\right| \geqslant 2 \delta$, one has $s \in e_{i}$ for some $i$ by definition of $e_{i}$, and $s \in e_{i}^{\prime}$ by $\left(2_{\alpha}\right)$; a fortiori, $s \in e_{i}^{\prime \prime}$, for $a_{i}<g\left(e_{i}^{\prime}\right)$ and $b_{i}>d\left(e_{i}^{\prime}\right)$. Hence $\operatorname{sgn} V_{s}^{\prime}=\operatorname{sgn} V_{s}$ by definition of the signs of $V^{\prime}$, and $\left|V_{s}^{\prime}-V_{s}\right|=|| V_{s}^{\prime}|-| V_{s}||<4 \delta<\varepsilon$ by $\left(1_{\delta}\right)$. On the opposite, if $\left|V_{s}\right|<2 \delta$, then $\left|V_{s}^{\prime}-V_{s}\right| \leqslant\left|V_{s}^{\prime}\right|+\left|V_{s}\right|<\left(\left|V_{s}\right|+4 \delta\right)+\left|V_{s}\right|<2 \delta+4 \delta+2 \delta<\varepsilon$.

The last case is the interval $] T \wedge t, t]$. There, $\tilde{U}=U^{\prime}$ and $|\tilde{U}-U|<\delta$; so

$$
\left|\inf _{[T, s]} \tilde{U}-\inf _{[T, s]} U\right|<\delta .
$$

Taking into account that $\tilde{U}>-3 \delta$ before $T$, we have $|\mathbf{I} \circ \tilde{U}-I|<4 \delta$ on $[0, t]$, and

$$
\left.\left.|| V^{\prime}|-| V||=|\mathbf{J} \circ \tilde{U}-(U-I)|=|(\tilde{U}-U)-(\mathbf{I} \circ \tilde{U}-I)|<5 \delta \quad \text { on }\right] T \wedge t, t\right] .
$$

As before, there are now two sub-cases. For an $s \in] T \wedge t, t]$ such that $\left|V_{s}\right| \geqslant \varepsilon / 4$, one has $s \in \ell_{j}$ for some $j$ by definition of $\ell_{j}$, and more precisely $s \in\left[g\left(\ell_{j}\right)+2 \delta, d\left(\ell_{j}\right)-2 \delta\right]$ owing to $\left(2_{\delta}\right)$. Consequently $s$ is also in the excursion $\ell_{j}^{\prime}$, and $\operatorname{sgn} V_{s}^{\prime}=\operatorname{sgn} V_{s}$; this implies $\left|V_{s}^{\prime}-V_{s}\right|=|| V_{s}^{\prime}|-| V_{s}||<5 \delta<\varepsilon$. If, on the opposite, $\left|V_{s}\right|<\varepsilon / 4$, then $\left|V_{s}^{\prime}-V_{s}\right| \leqslant\left|V_{s}^{\prime}\right|+\left|V_{s}\right|<\left(\left|V_{s}\right|+5 \delta\right)+\left|V_{s}\right|<\varepsilon / 4+5 \delta+\varepsilon / 4<\varepsilon$ by $\left(1_{\delta}\right)$. This establishes $\left(1_{\alpha}\right)$ and proves Proposition 4.8 .

\section{First PROPERTIES OF PARTIAL LÉVy RAISES}

As explained in Section 2, our goal is to establish topological recurrence of $\mathbf{T}$ via Corollary 2.4, by showing that each non-empty open subset of $\mathbb{W}$ is attainable. Exploiting Proposition 4.5, this section will show how attainability of open sets can be eased with the use of partial Lévy raises. 
For each $\eta>0$, the definition of attainablilty involves a finite number of Lévy raises. Thanks to Proposition 4.5, it is possible to use a random (possibly unbounded) number of partial Lévy raises in lieu of a deterministic number of Lévy raises. This is shown by the next proposition, whose proof will use the following notation: for $\varphi \in \mathbb{W}, t>0$ and $\varepsilon>0$, we define the tubular neighborhood of $\varphi$

$$
G_{t, \varepsilon}(\varphi)=\{w \in \mathbb{W}: \forall s \in[0, t] \quad|w(s)-\varphi(s)|<\varepsilon\} ;
$$

remark that $G_{t^{\prime}, \varepsilon^{\prime}}(\varphi) \subset G_{t, \varepsilon}(\varphi)$ if $t^{\prime} \geqslant t$ and $\varepsilon^{\prime} \leqslant \varepsilon$, and that the sequence $G_{n, \frac{1}{n}}(\varphi)$ is decreasing and forms a base of neighborhoods of $\varphi$.

Proposition 5.1. Given an open set $G \subset \mathbb{W}$, suppose that for every $\eta>0$ there exists an infinite sequence $\left(U^{0}, U^{1}, \ldots\right)$ of partial Lévy raises starting from a Brownian motion $U^{0}$ and such that $\mathbb{P}\left[\exists n \geqslant 0 \quad U^{n} \in G\right]>$ $1-\eta$. Then $G$ is attainable.

Proof. Given $G$ and $\eta$, the hypothesis yields a sequence $\left(U^{n}\right)_{n \geqslant 0}$ of processes; introduce the r.v. $N \leqslant \infty$ defined as the smallest $n$ such that $U^{n} \in G$. The hypothesis says that $\mathbb{P}[N=\infty]<\eta$; hence $\mathbb{P}[N>p]<2 \eta$ for some deterministic $p<\infty$ fixed in the sequel. The processes $X^{n}=U^{N \wedge n}$ satisfy

$$
\left(X^{n-1}, X^{n}\right)= \begin{cases}\left(U^{n-1}, U^{n}\right) & \text { on the event }\{N \geqslant n\} \\ \left(X^{n-1}, X^{n-1}\right) & \text { on the event }\{N<n\}\end{cases}
$$

so each pair $\left(X^{n-1}, X^{n}\right)$ is a partial Lévy raise (with a random time $T$ which is infinite on $\left.\{N<n\}\right)$. On the event $\{N \leqslant p\}$, whose probability is $>1-2 \eta$, one has $X^{p}=U^{N} \in G$, and hence also $G_{k, k^{-1}}\left(X^{p}\right) \subset G$ for all $k$ large enough; therefore it is possible to choose some deterministic $t>0$ and $\varepsilon>0$ so that $\mathbb{P}\left[G_{t, \varepsilon}\left(X^{p}\right) \subset G\right]>1-3 \eta$.

Apply now Proposition 4.5 to the sequence $\left(X^{0}, \ldots, X^{p}\right)$ of partial Lévy raises; this gives a sequence $\left(Y^{0}, \ldots, Y^{p}\right)$ of Lévy raises such that $Y^{p} \in G_{t, \varepsilon}\left(X^{p}\right)$ and $\left|Y^{0}\right|=\left|U^{0}\right|$ on $[0, t]$. Introduce a Brownian motion $B$ independent of $Y^{p}$, and consider the finite sequence of Lévy raises $\left(Z^{-1}, Z^{0}, \ldots, Z^{p}\right)$ defined by $Z^{n}=\mathbf{T}^{p-n} \circ \Gamma$ for $-1 \leqslant n \leqslant p$, with

$$
\Gamma= \begin{cases}Y^{p} & \text { on the interval }[0, t] \\ B-B_{t}+Y_{t}^{p} & \text { on the interval }[t, \infty[;\end{cases}
$$

observe that $\Gamma$ is tame (Lem. 3.9). On $[0, t]$, one has $Z^{n}=Y^{n}$ for all $n \in\{0, \ldots, p\}$, and hence also $\left|Z^{0}\right|=$ $\left|Y^{0}\right|=\left|U^{0}\right|$; as a consequence, $Z^{-1}=\mathbf{T} \circ U^{0}$ on $[0, t]$; this is a Brownian motion. On $[t, \infty[$, Lemma 3.9 says that $Z^{-1}$ is a Brownian motion independent of $Y^{p}$ and a fortiori of $\mathbf{T}^{p+1} \circ Y^{p}$, which equals $Z^{-1}$ on $[0, t]$. Finally, $\mathbf{T}^{p+1} \circ \Gamma=Z^{-1}$ is a Brownian motion.

From $\Gamma=Y^{p}$ on $[0, t]$ and $Y^{p} \in G_{t, \varepsilon}\left(X^{p}\right)$, one gets $\Gamma \in G_{t, \varepsilon}\left(X^{p}\right)$; consequently $\mathbb{P}[\Gamma \in G] \geqslant$ $\mathbb{P}\left[G_{t, \varepsilon}\left(X^{p}\right) \subset G\right]>1-3 \eta$, and $G$ is attainable by Proposition 2.5 .

This section ends with two lemmas, to be used only much later.

\section{Lemma 5.2.}

(a) Suppose that $t>0$ and that $(U, V)$ is a partial Lévy raise; call $] g, d[$ the interval of the excursion of $V$ which straddles $t$. There exists a random isometry $\Phi: \mathbb{R} \rightarrow \mathbb{R}$, with derivative $\operatorname{sgn} V_{t}$, such that $U_{s}=\Phi\left(V_{s}\right)$ for all $s$ in $[g, d]$;

(b) still for $t>0$, suppose that $\left(U^{0}, \ldots, U^{n}\right)$ is a finite sequence of partial Lévy raises. Call $] g_{i}, d_{i}[$ the interval of the excursion of $U^{i}$ which straddles t. There exists a random isometry $\Psi: \mathbb{R} \rightarrow \mathbb{R}$ such that $U_{s}^{0}=\Psi\left(U_{s}^{n}\right)$ for all $s$ in $\bigcap_{i=1}^{n}\left[g_{i}, d_{i}\right]$.

Proof.

(a) The random time $T$ such that $(U, V, T)$ is a partial Lévy raise satisfies $V_{T}=0$ if $T$ is finite, for $U_{T}=0$ and $V=U$ on $[0, T]$. Consequently, $T$ cannot belong to $] g, d[$. If $T \geqslant d$, then $U=V$ on $[g, d]$, and the conclusion 
holds with $\Phi$ the identity map. If $T \leqslant g$, then $U_{s}-\inf _{[T, s]} U=\left|V_{s}\right|$ does not vanish for $\left.s \in\right] g, d[$. Therefore $\inf _{[T, s]} U$ remains constant for $s$ in this interval; call $c$ this constant. Still for $\left.s \in\right] g, d[$, one has

$$
U_{s}=\left|V_{s}\right|+c=\left(\operatorname{sgn} V_{s}\right) V_{s}+c=\left(\operatorname{sgn} V_{t}\right) V_{s}+c .
$$

This proves (a), with $\Phi(x)=\left(\operatorname{sgn} V_{t}\right) x+c$; $\Phi^{n}$.

(b) it suffices to call $\Phi^{i}$ the random isometry such that $U^{i-1}=\Phi^{i}\left(U^{i}\right)$ on $\left[g_{i}, d_{i}\right]$, and to set $\Psi=\Phi^{1} \circ \Phi^{2} \circ \ldots \circ$

Lemma 5.3. Fix $t>0$ and let $(U, V)$ and $\left(U^{\prime}, V^{\prime}\right)$ be two Lévy raises and $\varepsilon>0$ a random variable. Suppose that $\left|V_{t}\right|>\varepsilon$ and, on the interval $[0, t]$,

$$
\left|V^{\prime}-V\right|<\varepsilon \quad \text { and } \quad\left|U^{\prime}-U\right|<\varepsilon .
$$

The random isometries $\Phi$ and $\Phi^{\prime}$ such that $U=\Phi(V)$ and $U^{\prime}=\Phi^{\prime}\left(V^{\prime}\right)$ in a (random) neighborhood of $t$ (see Lem. 5.2 (a)) satisfy

$$
\forall x \in \mathbb{R} \quad\left|\Phi^{\prime}(x)-\Phi(x)\right|<2 \varepsilon .
$$

Proof. From $\left|V_{t}\right|>\varepsilon$ and $\left|V_{t}^{\prime}-V_{t}\right|<\varepsilon$, one draws $\operatorname{sgn} V_{t}^{\prime}=\operatorname{sgn} V_{t}$. For $x \in \mathbb{R}$, writing

$$
\Phi^{\prime}(x)=\left(x-V_{t}^{\prime}\right) \operatorname{sgn} V_{t}^{\prime}+U_{t}^{\prime} \quad \text { and } \quad \Phi(x)=\left(x-V_{t}\right) \operatorname{sgn} V_{t}+U_{t}
$$

and using $\operatorname{sgn} V_{t}^{\prime}=\operatorname{sgn} V_{t}$ we obtain

$$
\left|\Phi^{\prime}(x)-\Phi(x)\right|=\left|\left(V_{t}-V_{t}^{\prime}\right) \operatorname{sgn} \Phi+U_{t}^{\prime}-U_{t}\right| \leqslant\left|V_{t}-V_{t}^{\prime}\right|+\left|U_{t}^{\prime}-U_{t}\right|<2 \varepsilon .
$$

\section{Planing a PATH}

Our final goal, proving attainability of $G$ by Proposition 5.1, is not yet within reach; some intermediate steps will be necessary. This section introduces a method which we call planing, for it eventually removes the relief of a path by repeatedly levelling its elevations.

Proposition 6.1. Suppose given two r.v. $t>0$ and $\varepsilon>0$, a tame process $U$ and a random time $T$ valued in $[0, \infty]$, such that $T$ is the end of an excursion of $U$ on the event $\{0<T<\infty\}$.

There exist an infinite sequence $\left(U^{0}, U^{1}, \ldots\right)$ of partial Lévy raises and a finite r.v. $N \geqslant 0$ such that

- $U^{0}=U$;

- each $U^{n}$ equals $U$ on the interval $[0, T]$;

- $\sup _{s \in[T, t]}\left|U_{s}^{N}\right|<\varepsilon$ on the event $\{T \leqslant t\}$.

This section is entirely devoted to proving Proposition 6.1.

Notice first that on the event $\{T \geqslant t\}$, the sequence defined by $U^{n}=U$ for all $n$ fulfills all required properties, since $(U, U)$ is a partial Lévy raise and $U_{T}=0$. So it suffices to construct the $U^{n}$ on the event $\{T<t\}$; at the cost of replacing $\mathbb{P}$ with a conditional probability, we may, and henceforth do, suppose that $T<t$ a.s.

Construction (planing). Our Proof of Proposition 6.1 will rely on the following construction, where $t, \varepsilon, U$ and $T$ are fixed and satisfy all hypotheses of Proposition 6.1, plus $T<t$. Fix also a series of r.v. $\left(\delta_{n}\right)$ such that $\delta_{n}>0$ and $\sum_{n \geqslant 1} \delta_{n}<\varepsilon$. Starting from $U^{0}=U$ and from $t_{0}=T$, construct a sequence $\left(U^{n}\right)_{n \geqslant 0}$ of processes and an increasing sequence $\left(t_{n}\right)_{n \geqslant 0}$ of random times in $[0, \infty]$ according to the following procedure.

Suppose $U^{n-1}$ and $t_{n-1}$ have already been constructed. Then

- $t_{n}$ is the first time after $t_{n-1}$ that $\mathbf{J}^{[T} U^{n-1}$ starts an excursion taller than $\varepsilon$;

- on $[0, T], U^{n}=U$; and on $\left[T, \infty\left[, U^{n}\right.\right.$ has absolute value $\left|U^{n}\right|=\mathbf{J}^{[T} U^{n-1}$;

- the excursion of $U^{n}$ starting at $t_{n}$ (which is taller than $\varepsilon$ ) is made negative; 
- all other excursions of $U^{n}$ taller than $\delta_{n}$ and started in the interval $\left[T, t_{n} \vee t\right]$ are made positive;

- the signs of all other excursions of $U^{n}$ (that is, all excursions smaller than $\delta_{n}$ and started in $\left[T, t_{n} \vee t\right]$, or started after $t_{n} \vee t$ ) are drawn at random, according to the fair coin-tossing law $\pi$, independently of everything already existing.

At each step of this procedure, the process $U^{n}$ so constructed is tame, $\left(J^{[T} U^{n}\right)^{[T}=\mathbf{J} \circ\left(U^{n[T}\right)$ is tame too, $t_{n}$ is finite, and $\left(U^{n-1}, U^{n}\right)$ is a partial Lévy raise. (These properties hold for $n=0$; they carry over from $n-1$ to $n$ owing to Prop. 4.3).

To demonstrate Proposition 6.1, it suffices to prove both following lemmas:

Lemma 6.2. For $n \geqslant 1$, on the interval $\left[T, t_{n}\right]$ one has

$$
-\delta_{n}<U^{n}<\varepsilon+\delta_{1}+\ldots+\delta_{n-1} .
$$

Lemma 6.3. Almost surely, $\sup _{n} t_{n}>t$.

Proof of Proposition 6.1 (Lems. 6.2 and 6.3 are admitted). We have already seen that $\left(U^{n}\right)$ is a sequence of partial Lévy raises, and the first two items in the conclusion of Proposition 6.1 are satisfied by construction. To check the third one, take a r.v. $N \geqslant 1$ such that $t_{N}>t$ (Lem. 6.3), and observe that, by Lemma $6.2,\left|U^{N}\right|<2 \varepsilon$ on $\left[T, t_{N}\right]$ and a fortiori on $[T, t]$. This proves Proposition 6.1 , with a trifling $2 \varepsilon$ instead of $\varepsilon$.

We still have to prove Lemmas 6.2 and 6.3. Remark that they involve only the processes $\left(U^{n}\right)^{[T}$ (what happens on $[0, T]$ is irrelevant), and that the construction of $U^{n}$ on $\left[T, \infty\left[\right.\right.$ and of $t_{n}$ necessitates only to know $U^{n-1}$ on $\left[T, \infty\left[\right.\right.$ and $t_{n-1}$. Consequently, at the cost of respectively replacing $U, U^{n}, t_{n}$ and $t$ with $U^{[T},\left(U^{n}\right)^{[T}$, $t_{n}-T$ and $t-T$, we may, and henceforth do, suppose that $T=0$. In that case, by Proposition 3.8, each pair $\left(U^{n-1}, U^{n}\right)$ is not only a partial Lévy raise, but a Lévy raise; so $U^{n-1}=\mathbf{T} \circ U^{n}$.

For the sake of further reference, we repeat below the planing algorithm, under the simplifying assumption $T=0$ now in force.

Start with $U^{0}=U$ and $t_{0}=0$. After $t_{n-1}$ and $U^{n-1}$ have been constructed, define $t_{n}$ and $U^{n}$ as follows.

- $t_{n}$ is the first time after $t_{n-1}$ that $\mathbf{J} \circ U^{n-1}$ starts an excursion taller than $\varepsilon$;

- $U^{n}$ has absolute value $\left|U^{n}\right|=\mathbf{J} \circ U^{n-1}$ (whence $U^{n-1}=\mathbf{T} \circ U^{n}$ );

- the excursion of $U^{n}$ started at $t_{n}$ (which is taller than $\varepsilon$ ) is made negative;

- all other excursions of $U^{n}$ taller than $\delta_{n}$ and started before $t_{n} \vee t$ are made positive;

- the signs of the excursions of $U^{n}$ smaller than $\delta_{n}$ or started after $t_{n} \vee t$ are drawn at random, according to a fair coin-tossing independent of everything already constructed.

We begin the proofs of the two lemmas. First, Lemma 6.2 ; it is not difficult.

Proof of Lemma 6.2. The minoration is obvious from the construction of $U^{n}$, whose first negative excursion taller than $\delta_{n}$ is the one starting at $t_{n}$. The majoration will be obtained by induction. Before $t_{1}$, all excursions of $\left|U^{1}\right|=\mathbf{J} \circ U^{0}$ are smaller than $\varepsilon$, so $U^{1}<\varepsilon$. Assume (induction hypothesis) that the claim holds for some $n \geqslant 1$. Call $u_{n}$ the first hitting time of $-\delta_{n}$ by $U^{n}$; this $u_{n}$ belongs to the excursion interval of $U^{n}$ originating at $t_{n}$ (because that excursion is taller than $\delta_{n}$ and negative, and $U^{n}>-\delta_{n}$ before $t_{n}$ ). On $\left[0, u_{n}\right]$, one has $-\delta_{n} \leqslant U^{n}<\varepsilon+\delta_{1}+\ldots+\delta_{n-1}$, whence also $\mathbf{J} \circ U^{n}<\varepsilon+\delta_{1}+\ldots+\delta_{n}$; and at time $u_{n}, U^{n}$ reaches its current minimum, so $\mathbf{J} \circ U^{n}\left(u_{n}\right)=0$. By definition of $t_{n+1}$, all excursions of $\mathbf{J} \circ U^{n}$ included in the interval $\left[t_{n}, t_{n+1}\right]$ are smaller than $\varepsilon$; hence $\mathbf{J} \circ U^{n}<\varepsilon$ on $\left[0, t_{n+1}\right] \backslash\left[0, u_{n}\right]$. So the majoration $\mathbf{J} \circ U^{n}<\varepsilon+\delta_{1}+\ldots+\delta_{n}$ on $\left[0, u_{n}\right]$ extends to $\left[0, t_{n+1}\right]$; and observing that $U^{n+1} \leqslant\left|U^{n+1}\right|=\mathbf{J} \circ U^{n}$ proves the claim for $n+1$.

The Proof of Lemma 6.3 is much less simple; it will occupy the rest of this section. It requires a new definition, which will be used in this section only. 
Definition 6.4. For $w \in \mathbb{W}, \alpha>0$ and $0 \leqslant a<k<b$, we say that the interval $[a, b]$ is an $\alpha$-arch of w, with keystone $k$, if $w(b)=w(a)=w(k)-\alpha$ and

$$
\forall t \in] a, k[\cup] k, b[\quad w(k)>w(t)>w(k)-\alpha,
$$

or if $w(b)=w(a)=w(k)+\alpha$ and

$$
\forall t \in] a, k[\cup] k, b[\quad w(k)<w(t)<w(k)+\alpha .
$$

In the first case, $w$ has a local maximum at $k$, and the arch is $u p ; a$ (resp. $b$ ) is the last time before $k$ (resp. the first time after $k$ ) when $w$ reaches the value $w(k)-\alpha$. A local maximum of $w$ is always the keystone of an $\alpha$-arch for every $\alpha$ small enough, but need not be the keystone of an $\alpha$-arch for an $\alpha$ given in advance.

In the other case, $w$ has a local minimum at $k$; the arch is down, and $[a, b]$ is also an $\alpha$-arch (which is up) of the opposite path $-w$, with the same keystone.

Clearly, the keystone of an $\alpha$-arch is unique.

When we speak of an $\alpha$-arch of a process, we mean a random $\alpha$-arch, possibly defined with probability less than $1 ; \alpha$ may be random too.

Lemma 6.5. Fix $w \in \mathbb{W}$ and $\alpha>0$.

(a) Given any $\alpha$-arch $A$ of $w$, for each $\beta$ such that $0<\beta \leqslant \alpha$ there exists a unique $\beta$-arch of $w$, included in $A$, and having the same keystone;

(b) given two different $\alpha$-arches of $w$, the keystone of any one of them cannot belong to the interior of the other one. The oscillation of $w$ on the interval between both keystones is at least $\alpha$;

(c) any two different $\alpha$-arches $A_{1}$ and $A_{2}$ of $w$ such that their interiors meet (i.e., $\left.\left(A_{1}\right)^{\circ} \cap\left(A_{2}\right)^{\circ} \neq \varnothing\right)$ have different orientations (one of them is up, the other down).

Proof of Lemma 6.5.

(a) Suppose that $A=[a, b]$ is up; then $w(k)=\max _{A} w$ and $w(a)=w(b)=w(k)-\alpha$. On each interval $] a, k[$ and $] k, b[, w$ assumes all values between $w(k)-\alpha$ and $w(k)$; so the numbers

$$
\begin{aligned}
a^{\prime} & =\sup \{t: t \leqslant k \text { and } w(t)=w(k)-\beta\} \\
b^{\prime} & =\inf \{t: t \geqslant k \text { and } w(t)=w(k)-\beta\}
\end{aligned}
$$

satisfy $a<a^{\prime}<k<b^{\prime}<b$ and $\left[a^{\prime}, b^{\prime}\right]$ is the $\beta$-arch of $w$ with keystone $k$. The case when $A$ is down is similar (or obtained by changing $w$ for $-w$ );

(b) to prove the first sentence, given an $\alpha$-arch $[a, b]$ with keystone $k$ and supposing that some $\left.k^{\prime} \in\right] a, b[$ is the keystone of an $\alpha$-arch $\left[a^{\prime}, b^{\prime}\right]$, we have to show that $\left[a^{\prime}, b^{\prime}\right]=[a, b]$. The oscillation of $w$ on any interval included in $] a, b[$ is less than $\alpha$; consequently,

$$
\begin{aligned}
a^{\prime} & =\sup \left\{t: t \leqslant k^{\prime} \text { and }\left|w(t)-w\left(k^{\prime}\right)\right|=\alpha\right\} \leqslant a, \\
b^{\prime} & =\inf \left\{t: t \geqslant k^{\prime} \text { and }\left|w(t)-w\left(k^{\prime}\right)\right|=\alpha\right\} \geqslant b,
\end{aligned}
$$

and therefore $\left[a^{\prime}, b^{\prime}\right] \supset[a, b]$. If $k^{\prime} \neq k, w\left(k^{\prime}\right)$ is not an extremal value of $w$ on $[a, b]$, nor a fortiori on $\left[a^{\prime}, b^{\prime}\right]$; this contradicts $k^{\prime}$ being the keystone of $\left[a^{\prime}, b^{\prime}\right]$. So $k^{\prime}=k$, and the above formulas yield $a^{\prime}=a$ and $b^{\prime}=b$ as claimed.

If $\left[a_{1}, b_{1}\right]$ and $\left[a_{2}, b_{2}\right]$ are two different $\alpha$-arches with respective keystones $k_{1}$ and $k_{2}$, we have $k_{1} \neq k_{2}$ (else $k_{1}$ would belong to $] a_{2}, b_{2}\left[\right.$ ); so suppose without loss of generality that $k_{1}<k_{2}$. Since $k_{1}$ does not belong to $] a_{2}, b_{2}$ [ but $k_{2}$ does, we have $k_{1}<a_{2}<k_{2}$. This entails $\operatorname{osc}_{\left[k_{1}, k_{2}\right]} w \geqslant \operatorname{osc}_{\left[a_{2}, k_{2}\right]} w=\alpha$;

(c) Let again $\left[a_{1}, b_{1}\right]$ and $\left[a_{2}, b_{2}\right]$ be two different $\alpha$-arches with keystones $k_{1}$ and $k_{2}$; we may suppose $k_{1}<k_{2}$. If $] a_{1}, b_{1}[\cap] a_{2}, b_{2}\left[\neq \varnothing\right.$, we have $a_{1}<k_{1}<a_{2}<b_{1}<k_{2}<b_{2}$. If the $\alpha$-arch $\left[a_{1}, b_{1}\right]$ is up, we must have $w\left(a_{2}\right)>w\left(b_{1}\right)$ and this implies that the $\alpha$-arch $\left[a_{2}, b_{2}\right]$ is down. The other case $\left(\left[a_{1}, b_{1}\right]\right.$ down $)$ is similar. 
Lemma 6.6. Let $t, \delta, \alpha$ and $\varepsilon$ be r.v. such that $t>0$ and $0<\delta<\alpha \leqslant \varepsilon$; let $V$ be a tame process all of whose negative excursions started before $t$ have amplitude $<\delta$, with the possible exception of at most one negative excursion with amplitude $\geqslant \varepsilon$.

Every $\alpha$-arch $A$ of $V$ included in $[0, t]$ contains an $(\alpha-\delta)$-arch $A^{\prime}$ of $\mathbf{T} \circ V$. On the event $\{A$ is up $\}, A^{\prime}$ can (and will) be chosen so as to have the same keystone as $A$.

If $A_{1}$ and $A_{2}$ are two $\alpha$-arches of $V$ included in $[0, t]$ with keystones $k_{1}$ and $k_{2}$ such that $k_{1}<k_{2}$, then the keystones $k_{1}^{\prime}$ and $k_{2}^{\prime}$ of $A_{1}^{\prime}$ and $A_{2}^{\prime}$ also satisfy $k_{1}^{\prime}<k_{2}^{\prime}$.

Proof of Lemma 6.6. Assume the hypotheses of the lemma, let $A=[a, b] \subset[0, t]$ be an $\alpha$-arch of $V$ with keystone $k$, and set $\beta=\alpha-\delta$. Put also $U=\mathbf{T} \circ V$.

We shall first consider the event $E=\left\{A\right.$ is down and $\left.-\delta<V_{k} \leqslant 0\right\}$. On this event, we have $V_{a}=V_{b}=$ $V_{k}+\alpha>-\delta+\alpha=\beta>0$, so $V$ must vanish somewhere on $\left[k, b\left[\right.\right.$; the last zero $k^{\prime}=\sup \left\{t \in A: V_{t}=0\right\}$ satisfies $k \leqslant k^{\prime}<b$. As the local time $L$ of $V$ is increasing, and constant on the excursions of $V, U_{k^{\prime}}$ is the minimum of $U=|V|-L$ on $[0, b]$ and a fortiori on $A$. Since

$$
U_{b}-U_{k^{\prime}}=\left|V_{b}\right|-L_{b}-\left|V_{k^{\prime}}\right|+L_{k^{\prime}}=V_{b}>\beta
$$

and

$$
U_{a}-U_{k^{\prime}}=\left|V_{a}\right|-L_{a}-\left|V_{k^{\prime}}\right|+L_{k^{\prime}} \geqslant V_{a}>\beta,
$$

$U$ has a $\beta$-arch $A^{\prime}$ with keystone $k^{\prime}$, and $A^{\prime}$ is included in $A$. This shows the existence of $A^{\prime}$ on the event $E$.

On the complementary event $E^{\mathrm{c}}$, we shall now show that the $\beta$-arch $A^{\prime}$ of $V$ with keystone $k$, which exists according to Lemma 6.5 (a), is also a $\beta$-arch of $U$ with the same keystone $k$. It suffices to verify that $V$ does not vanish on the interior $\left(A^{\prime}\right)^{\circ}$ of the interval $A^{\prime}$; indeed, in that case, the local time $L$ of $V$ is constant on $A^{\prime}$, and hence $A^{\prime}$, which is a $\beta$-arch of $V$, is also a $\beta$-arch of $U=|V|-L$.

To check that, on the event $E^{\mathrm{c}}, V$ does not vanish on $\left(A^{\prime}\right)^{\circ}$, we shall consider four cases.

Case 1. $A$ is down and $V_{k}>0$. As $V_{k}$ is the minimum of $V$ on $A, V$ cannot vanish on $A$, nor a fortiori on $A^{\prime}$.

Case 2. $A$ is down and $V_{k} \leqslant-\delta$. If $V_{a}$ were $>0$, the excursion of $V$ straddling $k$ would be included in the interior of $A$, so its amplitude would be $<\operatorname{osc}_{A} V=\alpha \leqslant \varepsilon$; this would contradict the hypothesis that no negative excursion of $V$ included in $[0, t]$ can have its amplitude in the range $\left[\delta, \varepsilon\left[\right.\right.$. So $V_{a} \leqslant 0$, and $A^{\circ}$, and a fortiori also $\left(A^{\prime}\right)^{\circ}$, is included in an excursion interval of $V$.

Case 3. $A$ is up and $V_{a}=V_{b}>-\delta$. We have $\inf _{A^{\prime}} V=V_{k}-\beta=V_{a}+\delta>0$, so $V$ cannot vanish on $A^{\prime}$.

Case 4. $A$ is up and $V_{a}=V_{b} \leqslant-\delta$. By hypothesis, $V$ has at most one negative excursion started before $t$ with amplitude $\geqslant \delta$; as $V_{a}=V_{b} \leqslant-\delta$, both $a$ and $b$ must belong to the same excursion interval of $V$, and the arches $A$ and $A^{\prime}$ are also included in this excursion interval.

This shows the first part of the lemma. Now, consider two $\alpha$-arches $A_{1}$ and $A_{2}$ of $V$ with respective keystones $k_{1}$ and $k_{2}$ such that $k_{1}<k_{2}$; and denote by $A_{1}^{\prime}$ and $A_{2}^{\prime}$ the corresponding $(\alpha-\delta)$-arches of $\mathbf{T} \circ V$, with keystones $k_{1}^{\prime}$ and $k_{2}^{\prime}$.

On the event $F=\left\{\left(A_{1}\right)^{\circ} \cap\left(A_{2}\right)^{\circ}=\varnothing\right\}$, each element of $\left(A_{1}\right)^{\circ}$ is anterior to each element of $\left(A_{2}\right)^{\circ}$, and in particular $k_{1}^{\prime}<k_{2}^{\prime}$.

On the complementary $F^{\mathrm{c}}=\left\{\left(A_{1}\right)^{\circ} \cap\left(A_{2}\right)^{\circ} \neq \varnothing\right\}$, Lemma 6.5 (c) says that one of $A_{1}$ and $A_{2}$ is up; so we split this event into two parts.

On $\left\{\left(A_{1}\right)^{\circ} \cap\left(A_{2}\right)^{\circ} \neq \varnothing\right.$ and $A_{1}$ is up $\}, A_{1}^{\prime}$ is chosen so that $k_{1}^{\prime}=k_{1}$. Since $k_{1}$ is anterior to $\left(A_{2}\right)^{\circ}$ by Lemma 6.5 (b) and $A_{2}^{\prime} \subset A_{2}$, we have $k_{1}^{\prime}<k_{2}^{\prime}$.

Similarly, on $\left\{\left(A_{1}\right)^{\circ} \cap\left(A_{2}\right)^{\circ} \neq \varnothing\right.$ and $A_{2}$ is up $\}, A_{2}^{\prime}$ is chosen so that $k_{2}^{\prime}=k_{2}$. Since $k_{2}$ is posterior to $\left(A_{1}\right)^{\circ}$ by Lemma 6.5 (b) and $A_{1}^{\prime} \subset A_{1}$, we also have $k_{1}^{\prime}<k_{2}^{\prime}$.

Proof of Lemma 6.3 (at last). We are given $t, \varepsilon$ and $\left(\delta_{n}\right)_{n \geqslant 1}$ such that $\sum_{n \geqslant 1} \delta_{n}<\varepsilon$; the $t_{n}$ and $U_{n}$ are constructed as described earlier. Choose $\alpha_{0}>0$ such that $\alpha_{0}+\sum_{n \geqslant 1} \delta_{n}<\varepsilon$, and set $\alpha_{n}=\alpha_{0}+\delta_{1}+\ldots+\delta_{n}<\varepsilon$. 
The first step of the proof consists in establishing that for $n \geqslant 1, t_{n+1}$ is the keystone of an $\alpha_{n}$-arch of $U^{n}$. Before $t_{n}, U^{n}$ is bounded below by $-\delta_{n}$ (Lem. 6.2). At time $t_{n}, U^{n}$ is null and starts a negative excursion $h_{n}$ exceeding $-\varepsilon$. Call $v_{n}$ the first time (equivalently, the first time after $t_{n}$ ) that $U^{n}$ hits $-\varepsilon$, and suppose $t_{n+1}<v_{n}$. Due to the definition of $t_{n+1}$, after $t_{n+1}, U^{n}$ must reach the value $U_{t_{n+1}}^{n}+\varepsilon$ before ever coming back to $U_{t_{n+1}}^{n}$. Since $U_{t_{n+1}}^{n}>-\varepsilon$ (because $t_{n+1}<v_{n}$ ), this implies that after $t_{n+1}, U^{n}$ reaches 0 before hitting $-\varepsilon$. This prevents the excursion $h_{n}$ to ever reach the value $-\varepsilon$, a contradiction; therefore our supposition that $t_{n+1}<v_{n}$ was false, and $t_{n+1} \geqslant v_{n}$. At time $t_{n+1}, U^{n}$ is at its current minimum because $\mathbf{J} \circ U^{n}=0$, so $U_{t_{n+1}}^{n} \leqslant U_{v_{n}}^{n}=-\varepsilon$. After $t_{n+1}, U^{n}$ reaches the value $U_{t_{n+1}}^{n}+\varepsilon$ before coming back to $U_{t_{n+1}}^{n}$. These properties entail that $t_{n+1}$ is the keystone of an $\varepsilon$-arch of $U^{n}$ (which is down), and a fortiori also of an $\alpha_{n}$-arch, since $\alpha_{n}<\varepsilon$.

The second step is the proof that for $n \geqslant 1$, the $\alpha_{n}$-arch of $U^{n}$ with keystone $t_{n+1}$ is included in $] t_{n}, t_{n+2}[$. Since $U_{t_{n}}^{n}=0$ and $U_{t_{n+1}}^{n} \leqslant-\varepsilon$, we have $U_{t_{n}}^{n}-U_{t_{n+1}}^{n} \geqslant \varepsilon>\alpha_{n}$, so $t_{n}$ does not belong to the $\alpha_{n}$-arch of $U^{n}$ with keystone $t_{n+1}$.

As $U_{t_{n+1}}^{n} \leqslant-\varepsilon, t_{n+1}$ belongs to the excursion $h_{n}$, and $U^{n}<0$ on $] t_{n}, t_{n+1}\left[\right.$. Hence the local time $L^{n}$ of $U^{n}$ is constant on this interval. But $U^{n-1}=\mathbf{T} \circ U^{n}=\left|U^{n}\right|-L^{n}$; so $U^{n-1}$ and $\left|U^{n}\right|$ have the same increment on ]$t_{n}, t_{n+1}\left[\right.$, and we have $U_{t_{n+1}}^{n-1}-U_{t_{n}}^{n-1}=\left|U_{t_{n+1}}^{n}\right|-\left|U_{t_{n}}^{n}\right|$. Observing that $U_{t_{n+1}}^{n} \leqslant-\varepsilon$ and $U_{t_{n}}^{n}=0$, we obtain $U_{t_{n+1}}^{n-1}-U_{t_{n}}^{n-1} \geqslant \varepsilon$, and changing $n$ for $n+1$ yields $U_{t_{n+2}}^{n}-U_{t_{n+1}}^{n} \geqslant \varepsilon$. A fortiori, $U_{t_{n+2}}^{n}-U_{t_{n+1}}^{n}>\alpha_{n}$; therefore $t_{n+2}$ cannot belong to the $\alpha_{n}$-arch of $U^{n}$ with keystone $t_{n+1}$.

We are now ready for the last step of the proof: supposing $\mathbb{P}\left[\forall n t_{n} \leqslant t\right]>0$, we shall reach a contradiction. By conditioning, we may suppose $t_{n} \leqslant t$ a.s. As the sequence $\left(t_{n}\right)$ is strictly increasing, we have $t_{n}<t$ for all $n$.

For $n \geqslant 1$, call $A_{n+1}^{n}$ the $\alpha_{n}$-arch of $U^{n}$ with keystone $t_{n+1}$ (step 1 ); by step $2, A_{n+1}^{n} \subset[0, t]$. We can repeatedly apply Lemma 6.6 and obtain a chain

$$
A_{n+1}^{n} \supset A_{n+1}^{n-1} \supset \ldots \supset A_{n+1}^{0},
$$

where $A_{n+1}^{i}$ is an $\alpha_{i}$-arch of $U^{i}$ included in $A_{n+1}^{i+1}$ and having the same keystone if $A_{n+1}^{i+1}$ is up. (To pass from $A_{n+1}^{i}$ to $A_{n+1}^{i-1}$, apply Lemma 6.6 with $V=U^{i}$, $\mathbf{T} \circ V=U^{i-1}, \alpha=\alpha_{i}, \delta=\delta_{i}$ and $\alpha-\delta=\alpha_{i-1}$. This is possible because $U^{i}$ is tame and $\mathbf{T} \circ U^{i}=U^{i-1}$, and all negative excursions of $U^{i}$ started in $[0, t]$ are smaller than $\delta_{i}$, except the one starting at $t_{i}$, which exceeds $-\varepsilon$ ). Call $k_{n+1}^{i}$ the keystone of $A_{n+1}^{i}$; for $n \geqslant 1$ we have $k_{n+1}^{n}=t_{n+1}$ by definition of $A_{n+1}^{n}$. Since $t_{n}$ is anterior to $A_{n+1}^{n}$ (step 2), $t_{n}$ is a fortiori anterior to $A_{n+1}^{n-1}$, and for $n \geqslant 2$ we have $k_{n}^{n-1}=t_{n}<k_{n+1}^{n-1}$. We can repeatedly apply Lemma 6.6 again, and successively obtain $k_{n}^{i}<k_{n+1}^{i}$ for all $i$, until finally $k_{n}^{0}<k_{n+1}^{0}$ for $n \geqslant 2$.

So we have a strictly increasing sequence $k_{2}^{0}<k_{3}^{0}<\ldots$ of keystones of $\alpha_{0}$-arches of $U^{0}$; all these arches are included in $[0, t]$, so $k_{n}^{0}<t$. By Lemma $6.5(\mathrm{~b})$, the oscillation of $U^{0}$ between $k_{n}^{0}$ and $k_{n+1}^{0}$ is $\geqslant \alpha_{0}$; this is incompatible with $U^{0}$ being continuous (or merely having left-limits), a contradiction. Lemma 6.3 and Proposition 6.1 are proven.

Remark 6.7. When proving Lemma 6.3, we have been a little lax and passed in silence over questions of measurability. In Lemma 6.6, we have not shown that $A^{\prime}$ can be measurably chosen, nor that the keystone of a measurable arch is measurable, etc. All this is of course true, and not difficult; but it turns out not to be necessary. All we need is the almost sure existence of $A^{\prime}$; the $k_{n}^{i}$ are only used to build up oscillation of $U^{0}$, and whether they are measurable or not is irrelevant.

\section{A Refinement of Proposition 6.1}

This section will prove Proposition 7.2, which improves in Proposition 6.1 by further specifying an approximate value for $U^{N}$ at time $t$. 
Lemma 7.1. Let $a, b$ and $t$ be three r.v. such that $0<a<b$ and $t>0$. If $U$ is a tame process, there exist finitely many different excursions $e_{1}, \ldots, e_{p}$ of $|U|$ included in $[0, t]$ (with $p$ random and the $e_{i}$ measurably chosen) such that, calling $h_{i}$ the amplitude of $e_{i}$, one has

$$
a<h_{1}+\ldots+h_{p}<b .
$$

Proof. We may suppose $U$ to be a Brownian motion. For arbitrary $s>0$ and $\varepsilon>0$, it is known that $U$ a.s. has infinitely many excursions included in $] 0, s[$ with amplitude less than $\varepsilon$; and the sum of all these amplitudes is infinite. The lemma follows by defining $e_{1}$ to be the tallest excursion of $|U|$ in $[0, t]$ with amplitude $h_{1}<b$, then $e_{2}$ to be the tallest among the remaining ones such that $h_{1}+h_{2}<b$, and so forth until the sum $h_{1}+\ldots+h_{p}$ overpasses $a$.

Proposition 7.2. Let $t>0$ be a r.v. and $I$ and $J$ two random, non-empty open intervals of the real line, such that $0 \in I$ and $J \subset I$. Let also $U$ be a tame process and $T$ a random time valued in $[0, \infty]$, such that $T$ is the end of an excursion of $U$ on the event $\{0<T<\infty\}$.

There exist an infinite sequence $\left(U^{n}\right)_{n \geqslant 0}$ of partial Lévy raises and a finite r.v. $N \geqslant 0$ such that

- $U^{0}=U$;

- each $U^{n}$ equals $U$ on the interval $[0, T]$;

- on the event $\{T \leqslant t\}, U_{s}^{N} \in I$ for all $s \in[T, t]$ and $U_{t}^{N} \in J$.

Proof. Replacing $I$ and $J$ with smaller intervals, we may without loss of generality suppose that, with probability 1 ,

$$
I=]-\varepsilon, a[\text { and } J=] a-\varepsilon, a[\quad \text { or } \quad I=]-a, \varepsilon[\text { and } J=]-a,-a+\varepsilon[
$$

for two r.v. $a$ and $\varepsilon$ such that $0<\varepsilon<a$.

We start by invoking Proposition 6.1 , to obtain $\left(U^{n}\right)_{n \geqslant 0}$ and $N$ such that $U^{0}=U, U^{n}=U$ on $[0, T]$ and $\sup _{s \in[T, t]}\left|U_{s}^{N}\right|<\varepsilon / 2$ on $\{T \leqslant t\}$. We shall keep constructing new partial Lévy raises from $U^{N}$ on; to avoid a cumbersome notation, it is convenient to set $V^{0}=U^{N}$ and to work on a sequence of partial Lévy raises $V^{n}$ (instead of $U^{N+n}$ ). The construction is only needed on the event $\{T<t\}$, so, by conditioning, we may suppose $T<t$ a.s. Also, all partial Lévy raises produced in the sequel of the proof will be prepared according to the recipe from Proposition 4.3 (with a $T$ in the recipe greater than or equal to the $T$ given in the statement of Prop. 7.2); working with $\left(V^{n}\right)^{[T}$ instead of $V^{n}$, Corollary 4.4 allows us to suppose $T=0$ without loss of generality. So we may (and henceforth do) suppose that $T=0$ and that $\left|V^{0}\right|<\varepsilon / 2$ on the interval $[0, t]$.

To construct $V^{1}$, we shall perform a (non partial) Lévy raise in accordance with Proposition 3.8, with all but finitely many excursion signs randomly chosen. The absolute value is given by $\left|V^{1}\right|=\mathbf{J} \circ V^{0} \leqslant\left|V^{0}\right|+\left|\mathbf{I} \circ V^{0}\right|$, so $\left|V^{1}\right|<\varepsilon$ on $[0, t]$. If $\left|V_{t}^{1}\right| \neq 0$, we choose the excursion straddling $t$ to be positive, whence $0 \leqslant V_{t}^{1}<\varepsilon$. As $\varepsilon<a$, Lemma 7.1 allows us to choose some excursions $e_{1}, \ldots, e_{p}$ of $\left|V^{1}\right|$ in the interval $[0, t]$ such that the sum $h_{1}+\ldots+h_{p}$ of their amplitudes belongs to the random interval $] a-V_{t}^{1}-\varepsilon, a-\varepsilon\left[\right.$. We number the $e_{i}$ in reverse chronological order: $e_{p}$ is the first one, $e_{1}$ the last one. Put

$$
\delta=\varepsilon \wedge h_{1} \wedge \ldots \wedge h_{p}
$$

and choose the signs of finitely many excursions of $V^{1}$ according to the following prescription: as already said, the excursion straddling $t$ is made positive; the $e_{i}$ are made negative; all other excursions before $t$ and with amplitude $\geqslant \delta$ are made positive.

Call $S_{i}$ the end of the excursion $e_{i}$, and put $S_{p+1}=0$.

For $1 \leqslant i \leqslant p$, we are now going to construct $V^{i+1}$ from $V^{i}$ by a partial Lévy raise with preservation of the past up to $S_{i+1}$. So $V^{i+1}=V^{i}$ on $\left[0, S_{i+1}\right]$, and $\left|V^{i+1}\right|=J^{\left[S_{i+1}\right.} V^{i}$ after $S_{i+1}$. Make positive the excursion of $V^{i+1}$ which straddles $t$ as well as all excursions included in $\left[S_{i+1}, t\right]$ with amplitude $\geqslant \delta$; and draw at random the signs of all other excursions of $V^{i+1}$ after $S_{i+1}$. By induction, we shall verify that, for $1 \leqslant i \leqslant p+1$, 
(1 $\left.1_{i}\right) V^{i}=V^{1}$ on $\left[0, S_{i}\right]$ (and so $S_{i+1}$ is the end of an excursion of $V^{i}$ for $i<p$ );

$\left(2_{i}\right) V^{i}>-\delta$ on $\left[S_{i}, t\right]$;

$\left(3_{i}\right) V^{i}<\varepsilon+h_{1}+\ldots+h_{i-1}$ on $[0, t]$

$\left(4_{i}\right)$ if $i \leqslant p$, the minimum of $V^{i}$ on the interval $\left[S_{i+1}, t\right]$ is $-h_{i}$ (attained during $e_{i}$ );

(5i) $V_{t}^{i}=V_{t}^{1}+h_{1}+\ldots+h_{i-1}$.

When $i=1,\left(1_{i}\right)$ and $\left(5_{i}\right)$ are trivial. The minoration $\left(2_{i}\right)$ holds for $V^{1}$ because the excursions of $V^{1}$ before $t$ and reaching or exceeding $\delta$ are positive, except the $e_{i}$, which are all anterior to $S_{1}$. The majoration $\left(3_{i}\right)$ is due to $V^{1} \leqslant\left|V^{1}\right|<\varepsilon$ on $[0, t]$. Last, in $\left[S_{2}, t\right], V^{1}$ has only one negative excursion with size $\geqslant \delta$, namely $e_{1}$, whence $\left(4_{i}\right)$.

Now, assuming these five properties to be true for $V^{i}$, we shall see that they also hold for $V^{i+1}$. First, before $S_{i+1}, V^{i+1}=V^{i}$ by preservation of the past, and in turn $V^{i}=V^{1}$ by $\left(1_{i}\right)$ because $S_{i+1}<S_{i}$. As all excursions $\geqslant \delta$ of $V^{i+1}$ in the interval $\left[S_{i+1}, t\right]$ are made positive, $\left(2_{i}\right)$ holds for $V^{i+1}$. The majoration $\left(3_{i}\right)$ for $V^{i+1}$ is verified separately on both intervals $\left[0, S_{i+1}\right]$ and $\left[S_{i+1}, t\right]$ : on the former, $V^{i+1}=V^{1}<\varepsilon$ by $\left(1_{i}\right)$, and, for $s \in\left[S_{i+1}, t\right]$, using the hypothesis $\left(4_{i}\right)$, one has the majoration

$$
V_{s}^{i+1} \leqslant\left|V_{s}^{i+1}\right|=V_{s}^{i}-\inf _{r \in\left[S_{i+1}, s\right]} V_{r}^{i} \leqslant V_{s}^{i}-\inf _{r \in\left[S_{i+1}, t\right]} V_{r}^{i}=V_{s}^{i}+h_{i},
$$

which transfers $\left(3_{i}\right)$ from $V^{i}$ to $V^{i+1}$. To check $\left(4_{i}\right)$ (when $i+1 \leqslant p$ ), we already have the minoration of $V^{i+1}$ by $-\delta$ on $\left[S_{i+1}, t\right]$ given by $\left(2_{i}\right)$; and on $\left[S_{i+2}, S_{i+1}\right], V^{i+1}$ equals $V^{1}$, whose only excursion in this interval attaining $-\delta$ is $e_{i+1}$; this shows $\left(4_{i}\right)$ for $V^{i+1}$. Last, since the excursion straddling $t$ is positive, using $\left(4_{i}\right)$ for $V^{i}$ one can write

$$
V_{t}^{i+1}=\left|V_{t}^{i+1}\right|=V_{t}^{i}-\inf _{s \in\left[S_{i+1}, t\right]} V_{s}^{i}=V_{t}^{i}+h_{i}
$$

so $V^{i+1}$ satisfies $\left(5_{i}\right)$ if $V^{i}$ does.

Observing now that $S_{p+1}=0$, the minoration $\left(2_{i}\right)$ yields $V^{p+1}>-\delta$ on $[0, t]$; and using the inequalities

$$
a-V_{t}^{1}-\varepsilon<h_{1}+\ldots+h_{p}<a-\varepsilon
$$

from the definition of the excursions $e_{i},\left(3_{i}\right)$ entails $V^{p+1}<a$ on $[0, t]$ and $\left(5_{i}\right)$ gives $a-\varepsilon<V_{t}^{p+1}<a+V_{t}^{1}-\varepsilon<a$. So if $I=]-\varepsilon, a[$ and $J=] a-\varepsilon, a\left[\right.$, we have obtained $V^{p+1} \in I$ on $[0, t]$ and $V_{t}^{p+1} \in J$. To also have these properties on the event

$$
E=\{I=]-a, \varepsilon[\text { and } J=]-a,-a+\varepsilon[\},
$$

it suffices to perform a further modification concerning the choice of the signs of $V^{p+1}$, which overrides the preceding prescription: on the event $E$, all excursions of $V^{p+1}$ started in $[0, t]$ and having amplitude $\geqslant \varepsilon \wedge(a-\varepsilon)$ are made negative (this is licit because the Lévy raise $\left(V^{p}, V^{p+1}\right)$ is not partial since $\left.S_{p+1}=0\right)$. As the absolute value $\left|V^{p+1}\right|$ is not changed by this new choice, one still has $\left|V^{p+1}\right|<a$ on $[0, t]$; the majoration $V^{p+1}<\varepsilon$ is obvious, and the estimate $-a<V_{t}^{p+1}<-a+\varepsilon$ holds because the sign of $V_{t}^{p+1}$ has been flipped to negative.

To achieve the proof of Proposition 7.2, it now suffices to define $V^{n}$ for $n>p+1$, by setting for instance $V^{n}=V^{p+1}$ for these $n$.

\section{FINAL STEPS}

Proposition 8.1. Let $G$ be a non-empty open subset of $\mathbb{W}$. For each $\eta>0$, there exists (on a suitable sample space) an infinite sequence $\left(U^{n}\right)_{n \geqslant 0}$ of partial Lévy raises such that $U^{0}$ is a Brownian motion and

$$
\mathbb{P}\left[U^{n} \in G \text { for some } n \geqslant 0\right]>1-\eta .
$$


This proposition is the only missing piece in our proof:

Theorem 8.2. The Lévy transformation is topologically recurrent: for almost all $w \in \mathbb{W}$, the orbit $\left\{\mathbf{T}^{n} w, n \geqslant 0\right\}$ is dense in $\mathbb{W}$.

Proof. Juxtapose Corollary 2.4, Propositions 5.1 and 8.1.

The rest of this section is the proof of Proposition 8.1.

For $w \in \mathbb{W}$ and $\Lambda \subset \mathbb{R}^{+}$, set $\|w\|_{\Lambda}=\sup _{t \in \Lambda}|w(t)|$.

For $\varphi \in \mathbb{W}, t>0$ and $\varepsilon>0$, recall that $G_{t, \varepsilon}(\varphi)$ denotes the tubular neighborhood $\left\{w \in \mathbb{W}:\|w-\varphi\|_{[0, t]}<\varepsilon\right\}$. These sets form a base for the topology of $\mathbb{W}$, hence the non-empty open set $G$ contains such a tubular neighborhood $G_{t, \varepsilon}(\varphi)$, and it suffices to prove Proposition 8.1 when $G=G_{t, \varepsilon}(\varphi)$.

From now on, $\varphi, t, \varepsilon$ and $\eta$ are fixed. By uniform continuity on $[0, t]$, there exists a number $p \geqslant 1$ such that

$$
\sup _{\substack{r, s \in[0, t] \\|r-s| \leqslant \frac{t}{p}}}|\varphi(r)-\varphi(s)|<\varepsilon
$$

and that, if $B$ is a Brownian motion,

$$
\mathbb{P}\left[\sup _{\substack{r, s \in[0, t] \\|r-s| \leqslant \frac{t}{p}}}\left|B_{r}-B_{s}\right|<\varepsilon\right]>1-\eta .
$$

Such a $p$ is fixed in the sequel. We also suppose that $\eta \leqslant 1 / 2$. This is of course quite harmless, but useful for it entails a further property of $p$ :

Lemma 8.3. Suppose $\eta \leqslant 1 / 2$. For any interval $\Lambda \subset \mathbb{R}^{+}$with length $t / p$, if $B$ is a Brownian motion one has

$$
\mathbb{P}\left[\sup _{r, s \in \Lambda}\left|B_{r}-B_{s}\right|<\varepsilon\right]>1-\frac{2 \eta}{p} .
$$

Proof of Lemma 8.3. The probability of the event $\Omega_{\Lambda}=\left\{\sup _{r, s \in \Lambda}\left|B_{r}-B_{s}\right|<\varepsilon\right\}$ depends only on the length of $\Lambda$, and, if $\Lambda_{q}=\left[\frac{(q-1) t}{p}, \frac{q t}{p}\right]$, the events $\Omega_{\Lambda_{1}}, \Omega_{\Lambda_{2}}, \ldots$ are independent. Clearly, the event

$$
\Omega^{\prime}=\left\{\sup _{\substack{r, s \in[0, t] \\|r-s| \leqslant \frac{t}{p}}}\left|B_{r}-B_{s}\right|<\varepsilon\right\}
$$

is included in $\bigcap_{q=1}^{p} \Omega_{\Lambda_{q}}$; and $\left(2_{p}\right)$ says that $\mathbb{P}\left[\Omega^{\prime}\right]>1-\eta$. Hence

$$
1-\eta<\mathbb{P}\left[\Omega^{\prime}\right] \leqslant \mathbb{P}\left[\bigcap_{q=1}^{p} \Omega_{\Lambda_{q}}\right]=\prod_{q=1}^{p} \mathbb{P}\left[\Omega_{\Lambda_{q}}\right]=\mathbb{P}\left[\Omega_{\Lambda_{1}}\right]^{p},
$$

wherefrom $\mathbb{P}\left[\Omega_{\Lambda_{1}}\right]>(1-\eta)^{\frac{1}{p}}$. To conclude, it suffices to check that

$$
\forall x \in\left[0, \frac{1}{2}\right] \quad(1-x)^{\frac{1}{p}} \geqslant 1-\frac{2 x}{p} .
$$

By concavity of $x \mapsto(1-x)^{\frac{1}{p}}$, it suffices to verify this inequality when $x=0$ (this case is trivial) and when $x=1 / 2$. In that case,

$$
(1-x)^{\frac{1}{p}}=(1 / 2)^{\frac{1}{p}}>(1 / \mathrm{e})^{\frac{1}{p}}>1-\frac{1}{p}=1-\frac{2 x}{p} .
$$


Our strategy for establishing Proposition 8.1 will consist in working sucessively on the time-intervals $\left[\frac{q t}{p}, \frac{(q+1) t}{p}\right]$. More precisely, we shall prove:

Claim 8.4. For each $q \in\{1, \ldots, p\}$, there exists an infinite sequence of partial Lévy raises $\left(U^{n}\right)_{n \geqslant 0}$ (depending on $q$ ) such that $U^{0}$ is a Brownian motion and that the event

$$
E_{q}=\left\{\text { for some } n, \quad\left\|U^{n}-\varphi\right\|_{\left[0, \frac{q}{p} t\right]}<3 \varepsilon \quad \text { and } \quad\left|U_{\frac{q}{p} t}^{n}-\varphi\left(\frac{q}{p} t\right)\right|<\varepsilon\right\}
$$

satisfies $\mathbb{P}\left[E_{q}\right]>1-4 \frac{q}{p} \eta$.

Taking $q=p$ and ignoring the second requirement in $E_{p}$, the claim immediately implies Proposition 8.1 (with $G=G_{t, 3 \varepsilon}(\varphi)$ and with $4 \eta$ instead of $\left.\eta\right)$.

Claim 8.4 will be proved by induction on $q$; so we start with $q=1$. As $\varphi(0)=0$, one has $\|\varphi\|_{\left[0, \frac{t}{p}\right]}<\varepsilon$ due to $\left(1_{p}\right)$. Proposition 7.2 with $U^{0}$ a Brownian motion, $\left.T=0, I=\right]-2 \varepsilon, 2 \varepsilon[$ and $J=] \varphi\left(\frac{t}{p}\right)-\varepsilon, \varphi\left(\frac{t}{p}\right)+\varepsilon[$, yields a sequence $\left(U^{n}\right)_{n \geqslant 0}$ of partial Lévy raises such that, for some random $N,\left\|U^{N}\right\|_{\left[0, \frac{t}{p}\right]}<2 \varepsilon$ and $\left|U_{\frac{t}{p}}^{N}-\varphi\left(\frac{t}{p}\right)\right|<\varepsilon$. It suffices to write $\left\|U^{N}-\varphi\right\|_{\left[0, \frac{t}{p}\right]} \leqslant\left\|U^{N}\right\|_{\left[0, \frac{t}{p}\right]}+\|\varphi\|_{\left[0, \frac{t}{p}\right]}<2 \varepsilon+\varepsilon$ to obtain the lemma for $q=1$ (with $\mathbb{P}\left[E_{1}\right]=1$ instead of $\left.\mathbb{P}\left[E_{1}\right]>1-4 \eta / p\right)$.

Supposing now the claim to be true for some $q$ such that $1 \leqslant q<p$, we are going to prove it for the next value $q+1$. So $q$ is fixed; we are given a sequence $\left(U^{n}\right)_{n \geqslant 0}$ of partial Lévy raises and a r.v. $N$ such that $U^{0}$ is a Brownian motion and the event

$$
E_{q}=\left\{\left\|U^{N}-\varphi\right\|_{\left[0, \frac{q t}{p}\right]}<3 \varepsilon \text { and }\left|U_{q t / p}^{N}-\varphi(q t / p)\right|<\varepsilon\right\}
$$

has probability $>1-4 \frac{q}{p} \eta$; our goal is to construct a similar sequence $\left(V^{n}\right)_{n \geqslant 0}$ with $q+1$ instead of $q$.

The instants $q t / p$ and $(q+1) t / p$ will play an important role in the proof; for typographical simplicity, we put

$$
t^{\prime}=\frac{q t}{p} \quad \text { and } \quad t^{\prime \prime}=\frac{(q+1) t}{p} .
$$

The first step is to modify the sequence $\left(U^{n}\right)$ by putting $U^{n}=U^{N}$ for all $n \geqslant N$. We still have a sequence of partial Lévy raises, and neither $U^{0}$ nor $E_{q}$ has changed; so from now on we suppose that $U^{n}=U^{N \wedge n}$. We can now pick a (deterministic) $m$ so that $\mathbb{P}[N \leqslant m]>1-\eta / p$. The event $E_{q}^{\prime}=E_{q} \cap\{N \leqslant m\}$ has probability $\mathbb{P}\left[E_{q}^{\prime}\right]>\mathbb{P}\left[E_{q}\right]-\eta / p$, and on $E_{q}^{\prime}$ we have $U^{m}=U^{N}$, whence

$$
\left\|U^{m}-\varphi\right\|_{\left[0, t^{\prime}\right]}<3 \varepsilon \text { and }\left|U_{t^{\prime}}^{m}-\varphi\left(t^{\prime}\right)\right|<\varepsilon .
$$

As both these inequalities are strict, there exists a r.v. $\varepsilon^{\prime}>0$ such that

$$
\text { on } E_{q}^{\prime}, \quad\left\|U^{m}-\varphi\right\|_{\left[0, t^{\prime}\right]}<3 \varepsilon-\varepsilon^{\prime} \quad \text { and } \quad\left|U_{t^{\prime}}^{m}-\varphi\left(t^{\prime}\right)\right|<\varepsilon-\varepsilon^{\prime} .
$$

Proposition 4.5 applied to $t^{\prime}, \varepsilon^{\prime}$ and $\left(U^{0}, U^{1}, \ldots, U^{m}\right)$ yields a sequence $\left(X^{0}, X^{1}, \ldots, X^{m}\right)$ of (non partial) Lévy raises such that, on $\left[0, t^{\prime}\right],\left|X^{0}\right|=\left|U^{0}\right|$ and $\left|X^{m}-U^{m}\right|<\varepsilon^{\prime}$. With an error at most $\varepsilon^{\prime}$, we can replace $U^{m}$ with $X^{m}$ in $(*)$, so

$$
\text { on } E_{q}^{\prime}, \quad\left\|X^{m}-\varphi\right\|_{\left[0, t^{\prime}\right]}<3 \varepsilon \text { and } \quad\left|X_{t^{\prime}}^{m}-\varphi\left(t^{\prime}\right)\right|<\varepsilon \text {. }
$$

Putting $X^{-1}=\mathbf{T} \circ X^{0}$, the sequence $\left(X^{-1}, X^{0}, \ldots, X^{m}\right)$ is also a sequence of Lévy raises; and on the interval $\left[0, t^{\prime}\right]$ the process $X^{-1}$ equals the Brownian motion $\mathbf{T} \circ U^{0}$ because $\left|X^{0}\right|=\left|U^{0}\right|$ on this interval. 
The next step is to take some Brownian motion $B^{m}$ independent from $X^{m}$, and to modify $X^{m}$ after time $t^{\prime}$ by setting

$$
Y^{m}=X^{m} \quad \text { on } \quad\left[0, t^{\prime}\right] ; \quad Y^{m}=X_{t^{\prime}}^{m}+B^{m}-B_{t^{\prime}}^{m} \quad \text { on } \quad\left[t^{\prime}, \infty[;\right.
$$

$Y^{m}$ also satisfies $\left(1_{\eta}\right)$. Then define a sequence $\left(Y^{-1}, Y^{0}, \ldots, Y^{m}\right)$ of Lévy raises by $Y^{n}=\mathbf{T}^{m-n} \circ Y^{m}$ and observe that $Y^{n}=X^{n}$ on $\left[0, t^{\prime}\right]$. Since $X^{-1}$ was a Brownian motion on $\left[0, t^{\prime}\right]$, Lemma 3.9 (b) says that $Y^{-1}$ is obtained by concatenating two independent Brownian motions; so $Y^{-1}$ is a Brownian motion. As for $Y^{m}$, its increments after $t^{\prime}$ are Brownian; consequently, since $t^{\prime \prime}-t^{\prime}=t / p$, Lemma 8.3 says that

$$
\sup _{r, s \in\left[t^{\prime}, t^{\prime \prime}\right]}\left|Y_{r}^{m}-Y_{s}^{m}\right|<\varepsilon \quad \text { with probability }>1-\frac{2 \eta}{p} .
$$

Extend the sequence $\left(Y^{-1}, Y^{0}, \ldots, Y^{m}\right)$ of Lévy raises indefinitely to the left by putting $Y^{-n}=\mathbf{T}^{n} \circ Y^{0}=$ $\mathbf{T}^{n-1} \circ Y^{-1}$ for all $n>1$. These processes $Y^{-n}$ are the iterated Lévy transforms of the Brownian motion $Y^{-1}$. It is shown in [2] that the set of all zeros of all these Brownian motions is a.s. dense in $\mathbb{R}^{+}$; so the r.v.

$$
\inf \left\{n \geqslant 1: Y^{-n} \text { has a zero in the interval }\left[t^{\prime}, t^{\prime \prime}\right]\right\}
$$

is a.s. finite, and there exists a deterministic $k>1$ such that, with probability $>1-\eta / p$,

$$
Y_{s}^{-n}=0 \text { for some } s \in\left[t^{\prime}, t^{\prime \prime}\right] \text { and some } n \in\{1, \ldots, k-1\} .
$$

The finite sequence of Lévy raises

$$
\left(Y^{-k}, Y^{-k+1}, \ldots, Y^{-1}, Y^{0}, \ldots, Y^{m}\right)
$$

starts with the Brownian motion $Y^{-k}=\mathbf{T}^{k-1} Y^{-1}$ and has property $\left(1_{\eta}\right)$ (with $Y^{m}$ instead of $X^{m}$ ) with probability $>\mathbb{P}\left[E_{q}\right]-\eta / p$, property $\left(2_{\eta}\right)$ with probability $>1-2 \eta / p$ and property $\left(3_{\eta}\right)$ with property $>1-\eta / p$. All in all, the event $E_{q+1}$ that these three properties hold simultaneously has probability

$$
\mathbb{P}\left[E_{q+1}\right]>\mathbb{P}\left[E_{q}\right]-\frac{\eta}{p}-\frac{2 \eta}{p}-\frac{\eta}{p}>\left(1-\frac{4 q \eta}{p}\right)-\frac{4 \eta}{p}=1-\frac{4(q+1) \eta}{p} ;
$$

this is exactly the estimate required in the induction argument. So Claim 8.4 will be proven if we exhibit a sequence $\left(V^{n}\right)_{n \geqslant 0}$ of partial Lévy raises started from $V^{0}=Y^{-k}$ and a r.v. $N \geqslant 0$ such that, on the event $E_{q+1}$, one a.s. has

$$
\left\|V^{N}-\varphi\right\|_{\left[0, t^{\prime \prime}\right]}<3 \varepsilon \text { and }\left|V_{t^{\prime \prime}}^{N}-\varphi\left(t^{\prime \prime}\right)\right|<\varepsilon
$$

The sequence $\left(V^{n}\right)$ will be obtained by modifying the $Y^{i}$; since we are only interested in the behavior of the $V^{n}$ on $E_{q+1}$, we may for instance put $V^{n}=Y^{-k}$ for all $n$ on $E_{q+1}^{\mathrm{c}}$, and concentrate on the event $E_{q+1}$. Technically it is convenient to do this by replacing the probability $\mathbb{P}$ with the conditional probability $\mathbb{P}\left[\quad \mid E_{q+1}\right]$. Of course, this also modifies the laws of the processes, but this change is harmless, because we no longer require $V^{0}$ to be a Brownian motion, we simply demand that $V^{0}=Y^{-k}$.

To ease the notations, we rename as $\left(Z^{0}, \ldots, Z^{\ell}\right)$ the sequence of Lévy raises $\left(Y^{-k}, \ldots, Y^{m}\right)$; since from here on we are working with a probability giving $E_{q+1}$ full measure, the following three properties now hold almost surely:

$$
\begin{gathered}
\left\|Z^{\ell}-\varphi\right\|_{\left[0, t^{\prime}\right]}<3 \varepsilon ; \\
\sup _{s \in\left[t^{\prime}, t^{\prime \prime}\right]}\left|Z_{s}^{\ell}-\varphi\left(t^{\prime}\right)\right|<2 \varepsilon ;
\end{gathered}
$$

$$
Z_{S}^{K}=0 \text { for some (random) } K \in\{1, \ldots, \ell\} \text { and } S \in\left[t^{\prime}, t^{\prime \prime}\right] .
$$

The first one comes from $\left(1_{\eta}\right)$, the second one from $\left(1_{\eta}\right)$ and $\left(2_{\eta}\right)$, and the third one from $\left(3_{\eta}\right)$. In $\left(3_{Z}\right)$, we choose $K$ to be maximal: for $n \in\{K+1, \ldots, \ell\}, Z^{n}$ does not vanish on $\left[t^{\prime}, t^{\prime \prime}\right]$. 
The inequalities in $\left(1_{Z}\right)$ and $\left(2_{Z}\right)$ are strict; and $Z_{t^{\prime}}^{n} \neq 0$ by tameness for each $n \in\{0, \ldots, \ell\}$. So there exists a r.v. $\varepsilon_{\ell}$ such that $0<\varepsilon_{\ell} \leqslant \varepsilon / 3$ and

$$
\begin{gathered}
\left\|Z^{\ell}-\varphi\right\|_{\left[0, t^{\prime}\right]}<3 \varepsilon-\varepsilon_{\ell} ; \\
\sup _{s \in\left[t^{\prime}, t^{\prime \prime}\right]}\left|Z_{s}^{\ell}-\varphi\left(t^{\prime}\right)\right|<2 \varepsilon-3 \varepsilon_{\ell} ; \\
\forall n \in\{0, \ldots, \ell\} \quad\left|Z_{t^{\prime}}^{n}\right|>\varepsilon_{\ell} .
\end{gathered}
$$

In Section 4 (partial Lévy raises), $\alpha$-doubles of a tame process were defined and used; a fixed time $t$ was implied. We shall deal with $\alpha$-doubles anew in the sequel, but from now on the fixed time understood in the definition of doubles is $t^{\prime}=q t / p$.

Applying the second part of Proposition 4.8 to the Lévy raise $\left(Z^{\ell-1}, Z^{\ell}\right)$, we can choose a r.v. $\varepsilon_{\ell-1}>0$ with the property that, for every $\varepsilon_{\ell-1}$-double $U$ of $Z^{\ell-1}$, there exists an $\varepsilon_{\ell^{-}}$double $V$ of $Z^{\ell}$ such that $(U, V)$ is a Lévy raise. Applying it again to $\left(Z^{\ell-2}, Z^{\ell-1}\right)$, then to $\left(Z^{\ell-3}, Z^{\ell-2}\right)$, and so forth all the way down to $\left(Z^{0}, Z^{1}\right)$, there exists a finite sequence $\left(\varepsilon_{0}, \ldots, \varepsilon_{\ell}\right)$ of strictly positive r.v. such that if $U$ is any $\varepsilon_{n}$-double of $Z^{n}$, there exists a $\varepsilon_{n+1}$-double $V$ of $Z^{n+1}$ making $(U, V)$ a Lévy raise. Moreover, since at each step $\varepsilon_{n}$ can be chosen arbitrarily small, we may (and do) further require

$$
\begin{gathered}
\text { for } 0 \leqslant n<\ell \quad \varepsilon_{n} \leqslant \varepsilon_{n+1} ; \\
2\left(\varepsilon_{1}+\ldots+\varepsilon_{\ell-1}\right)<\varepsilon_{\ell} .
\end{gathered}
$$

By Lemma $5.2(\mathrm{~b})$, for $0 \leqslant i \leqslant j \leqslant \ell$ there exists a random isometry $\Phi^{j \rightarrow i}: \mathbb{R} \rightarrow \mathbb{R}$ such that $\Phi^{j \rightarrow i}\left(Z^{j}\right)=Z^{i}$ in a (random) neighborhood of $t^{\prime}$; one has $\Phi^{j \rightarrow i} \circ \Phi^{k \rightarrow j}=\Phi^{k \rightarrow i}$. Put $a_{n}=\Phi^{\ell \rightarrow n}\left(\varphi\left(t^{\prime}\right)\right)$ and $b_{n}=\Phi^{\ell \rightarrow n}\left(\varphi\left(t^{\prime \prime}\right)\right)$ and define some open intervals

$$
\begin{aligned}
& \left.\quad I_{n}=\right] a_{n}-\alpha_{n}, a_{n}+\alpha_{n}\left[, \quad \text { with } \alpha_{n}=2 \varepsilon-2\left(\varepsilon_{n+1}+\ldots+\varepsilon_{\ell}\right)\right. \\
& \text { and } \left.\quad J_{n}=\right] b_{n}-\beta_{n}, b_{n}+\beta_{n}\left[, \quad \text { with } \beta_{n}=\varepsilon-2\left(\varepsilon_{n+1}+\ldots+\varepsilon_{\ell}\right) ;\right.
\end{aligned}
$$

observe that $\alpha_{n}>\beta_{n}>0$ by $\left(5_{\varepsilon}\right)$ and because $3 \varepsilon_{\ell} \leqslant \varepsilon$; so $I_{n}$ and $J_{n}$ are not empty. Observe also that $I_{n} \supset J_{n}$ because, owing to $\left(1_{p}\right)$,

$$
\left|b_{n}-a_{n}\right|=\left|\Phi^{\ell \rightarrow n}\left(\varphi\left(t^{\prime \prime}\right)\right)-\Phi^{\ell \rightarrow n}\left(\varphi\left(t^{\prime}\right)\right)\right|=\left|\varphi\left(t^{\prime \prime}\right)-\varphi\left(t^{\prime}\right)\right|<\varepsilon=\alpha_{n}-\beta_{n}
$$

Notation 8.5. If $Q$ is any process, we write $Q_{[a, b]}$ for the process $\left(Q_{s}\right)_{a \leqslant s \leqslant b}$, and if $I$ is some interval, $Q_{[a, b]} \subset I$ means that $Q_{s} \in I$ for all $s \in[a, b]$.

To terminate the proof of Claim 8.4 (and hence also of the theorem), it suffices to demonstrate the following claim, where $K$ is the maximal random index satisfying $\left(3_{Z}\right)$ and where the fixed time understood in the definition of a double is $t^{\prime}$.

Claim 8.6. There exists an infinite sequence $\left(V^{i}\right)_{i \geqslant 0}$ of partial Lévy raises and a finite sequence $\left(M_{0}, M_{1}, \ldots, M_{\ell}\right)$ of integer-valued r.v. such that $M_{0}<M_{1}<\ldots<M_{\ell}$ and, for each $n \in\{0, \ldots, \ell\}$,

(a) $V^{M_{n}}$ is an $\varepsilon_{n}$-double of $Z^{n}$;

(b) on the event $\{n<K\}$ one has $M_{n}=n$ and $V^{n}=Z^{n}$;

(c) on the event $\{n \geqslant K\}$ one has $V_{\left[t^{\prime}, t^{\prime \prime}\right]}^{M_{n}} \subset I_{n}$ and $V_{t^{\prime \prime}}^{M_{n}} \in J_{n}$. 
Proof that Claim 8.6 $\Rightarrow$ Claim 8.4. We have $V^{0}=Z^{0}$ by Claim 8.6 (b) owing to $K>0$; so it suffices to check that (**) holds. Taking $N=M_{\ell}$, one has $\left\|V^{N}-Z^{\ell}\right\|_{\left[0, t^{\prime}\right]}<\varepsilon_{\ell}$ by (a); so $\left(1_{\varepsilon}\right)$ implies that

$$
\left\|V^{N}-\varphi\right\|_{\left[0, t^{\prime}\right]}<\varepsilon_{\ell}+\left(3 \varepsilon-\varepsilon_{\ell}\right)=\varepsilon .
$$

On remarking that $a_{\ell}=\varphi\left(t^{\prime}\right), b_{\ell}=\varphi\left(t^{\prime \prime}\right), \alpha_{\ell}=2 \varepsilon$ and $\beta_{\ell}=\varepsilon$, Claim 8.6 (c) gives

$$
\left\|V^{N}-\varphi\left(t^{\prime}\right)\right\|_{\left[t^{\prime}, t^{\prime \prime}\right]}<2 \varepsilon \quad \text { and } \quad\left|V_{t^{\prime \prime}}^{N}-\varphi\left(t^{\prime \prime}\right)\right|<\varepsilon
$$

together with $\left\|\varphi-\varphi\left(t^{\prime}\right)\right\|_{\left[t^{\prime}, t^{\prime \prime}\right]}<\varepsilon$, which is a consequence of $\left(1_{p}\right)$, the $2 \varepsilon$-estimate yields $\left\|V^{N}-\varphi\right\|_{\left[t^{\prime}, t^{\prime \prime}\right]}<3 \varepsilon$, and $(* *)$ is proven.

It remains to establish Claim 8.6; the a.s. finite sequence $\left(V^{0}, \ldots, V^{M_{n}}\right)$ will be constructed by induction on $n$. By (b), we must start with $M_{0}=0$ and $V^{0}=Z^{0}$. For some $n<\ell$ assume that $M_{0}, \ldots, M_{n}$ and $V^{0}, \ldots, V^{M_{n}}$ have already been defined and satisfy (a), (b) and (c); we must construct $M_{n+1}>M_{n}$ and $V^{M_{n}+1}, \ldots, V^{M_{n+1}}$ so that $\left(V^{M_{n}}, V^{M_{n}+1}, \ldots, V^{M_{n+1}}\right)$ is a sequence of partial Lévy raises (with random length) and so that (a), (b) and (c) hold for $n+1$ too.

Writing for simplicity $L=M_{n+1}-M_{n}$ and

$$
\left(R^{0}, \ldots, R^{L}\right)=\left(V^{M_{n}}, V^{M_{n}+1}, \ldots, V^{M_{n+1}}\right),
$$

it suffices to prove the next claim, where $n<\ell$ is fixed.

Claim 8.7. Let $R^{0}$ be a process such that

(a') $R^{0}$ is an $\varepsilon_{n}$-double of $Z^{n}$;

(b') $R^{0}=Z^{n}$ on $\{n<K\}$;

(c') $R_{\left[t^{\prime}, t^{\prime \prime}\right]}^{0} \subset I_{n}$ and $R_{t^{\prime \prime}}^{0} \in J_{n}$ on $\{n \geqslant K\}$.

There exists a sequence $\left(R^{i}\right)_{i \geqslant 0}$ of partial Lévy raises, started from this $R^{0}$, and an integer-valued r.v. $L$ such that

(a") $R^{L}$ is an $\varepsilon_{n+1}$-double of $Z^{n+1}$;

(b") $L=1$ and $R^{1}=Z^{n+1}$ on $\{n+1<K\}$;

(c") $R_{\left[t^{\prime}, t^{\prime \prime}\right]}^{L} \subset I_{n+1}$ and $R_{t^{\prime \prime}}^{L} \in J_{n+1}$ on $\{n+1 \geqslant K\}$.

Proof of Claim 8.7. Three cases will be distinguished.

(1) On the event $\{n+1<K\}, L=1$ and $R^{i}=Z^{n+1}$ for all $i \geqslant 1$ meet the requirements (a") and (b"); (c") is not relevant;

(2) on the event $\{n+1=K\}, R^{0}=Z^{n}$ by (b'). Set $R^{1}=Z^{n+1}=Z^{K}$. By definition of $K$ (see $\left(3_{Z}\right)$ ), there exists $s \in\left[t^{\prime}, t^{\prime \prime}\right]$ such that $R_{s}^{1}=0$. By maximality of $K$, for $n+1<i \leqslant \ell, Z^{i}$ does not vanish on $\left[t^{\prime}, t^{\prime \prime}\right]$; hence, according to Lemma $5.2(\mathrm{~b}), R_{s}^{1}=\Phi^{\ell \rightarrow n+1}\left(Z_{s}^{\ell}\right)$ for all $s \in\left[t^{\prime}, t^{\prime \prime}\right]$. As $\Phi^{\ell \rightarrow n+1}$ is an isometry, using $\left(2_{\varepsilon}\right)$ and $\left(5_{\varepsilon}\right)$ one can write for $s \in\left[t^{\prime}, t^{\prime \prime}\right]$

$$
\begin{aligned}
\left|R_{s}^{1}-a_{n+1}\right| & =\left|\Phi^{\ell \rightarrow n+1}\left(Z_{s}^{n+1}\right)-\Phi^{\ell \rightarrow n+1}\left(\varphi\left(t^{\prime}\right)\right)\right| \\
& =\left|Z_{s}^{n+1}-\varphi\left(t^{\prime}\right)\right|<2 \varepsilon-3 \varepsilon_{\ell}<\alpha_{0}<\alpha_{n+1}
\end{aligned}
$$

this shows that $R_{\left[t^{\prime}, t^{\prime \prime}\right]}^{1} \subset I_{n+1}$. Call $T$ the first zero of $R^{1}$ on $\left[t^{\prime}, t^{\prime \prime}\right]$; since $0=R_{T}^{1}$ is one of the values of $R^{1}$ on $\left[t^{\prime}, t^{\prime \prime}\right]$, it belongs to $I_{n+1}$. We may apply Proposition 7.2 to $t^{\prime \prime}, I_{n+1}, J_{n+1}, R^{1}$ and $T$, and we obtain a sequence $\left(R^{1}, R^{2}, \ldots\right)$ of partial Lévy raises such that each $R^{i}$ equals $R^{1}$ on $[0, T]$, and such that for some $L \geqslant 1$ one has $R_{\left[T, t^{\prime \prime}\right]}^{L} \subset I_{n+1}$ and $R_{t^{\prime \prime}}^{L} \in J_{n+1}$. (a") is satisfied because $R^{L}=R^{1}=Z^{n+1}$ on $\left[0, t^{\prime}\right]$, and (c") is satisfied too because $R_{\left[t^{\prime}, T\right]}^{L}=R_{\left[t^{\prime}, T\right]}^{1} \subset I_{n+1}$; 
(3) on the event $\{n+1>K\}$, one has $n \geqslant K$, so by (c') $R_{\left[t^{\prime}, t^{\prime \prime}\right]}^{0} \subset I_{n}$ and $R_{t^{\prime \prime}}^{0} \in J_{n}$. According to (a'), $R^{0}$ is an $\varepsilon_{n}$-double of $Z^{n}$; by definition of $\varepsilon_{n}$ (and by the second part of Prop. 4.8), there exists $R^{1}$, an $\varepsilon_{n+1}$-double of $Z^{n+1}$, such that $\left(R^{0}, R^{1}\right)$ is a Lévy raise.

Call $T$ the first zero of $R^{1}$ on $\left[t^{\prime}, \infty\left[\right.\right.$, and $\Phi^{\prime}$ the random isometry given by Lemma $5.2(\mathrm{a})$, such that $\Phi^{\prime}\left(R^{1}\right)=R^{0}$ on $\left[t^{\prime}, T\right]$. By $\left(3_{\varepsilon}\right)$ and $\left(4_{\varepsilon}\right),\left|Z_{t^{\prime}}^{n}\right|>\varepsilon_{\ell} \geqslant \varepsilon_{n+1}$; by $\left(4_{\varepsilon}\right), \varepsilon_{n} \leqslant \varepsilon_{n+1}$. We may apply Lemma 5.3 to $t^{\prime},\left(Z^{n}, Z^{n+1}\right)$, $\left(R^{0}, R^{1}\right)$ and $\varepsilon_{n+1}$ (in lieu of $t,(U, V),\left(U^{\prime}, V^{\prime}\right)$ and $\varepsilon$ in the notation of the lemma); we obtain

$$
\forall x \quad\left|\Phi^{\prime}(x)-\Phi^{n+1 \rightarrow n}(x)\right| \leqslant 2 \varepsilon_{n+1} .
$$

Thus, for all $s \in\left[t^{\prime}, T \wedge t^{\prime \prime}\right]$,

$$
\begin{aligned}
\left|R_{s}^{1}-a_{n+1}\right| & =\left|\Phi^{\prime}\left(R_{s}^{1}\right)-\Phi^{\prime}\left(a_{n+1}\right)\right|=\left|R_{s}^{0}-\Phi^{\prime}\left(a_{n+1}\right)\right| \\
& \leqslant\left|R_{s}^{0}-\Phi^{n+1 \rightarrow n}\left(a_{n+1}\right)\right|+\left|\Phi^{n+1 \rightarrow n}\left(a_{n+1}\right)-\Phi^{\prime}\left(a_{n+1}\right)\right| \\
& <\left|R_{s}^{0}-a_{n}\right|+2 \varepsilon_{n+1} .
\end{aligned}
$$

As $\left|R_{s}^{0}-a_{n}\right|<\alpha_{n}$ by hypothesis (c'), we obtain

$$
\left|R_{s}^{1}-a_{n+1}\right|<\alpha_{n}+2 \varepsilon_{n+1}=\alpha_{n+1},
$$

that is,

$$
R_{\left[t^{\prime}, T \wedge t^{\prime \prime}\right]}^{1} \subset I_{n+1} .
$$

To end the proof, there are now two sub-cases. On the event $\left\{T \geqslant t^{\prime \prime}\right\}$, we will take $L=1$. Condition (a") is satisfied because $R^{1}$ was defined as an $\varepsilon_{n+1}$-double of $Z^{n+1}$; we now check (c"). Inclusion (***) implies $R_{\left[t^{\prime}, t^{\prime \prime}\right]}^{1} \subset I_{n+1}$, and it only remains to show that $R_{t^{\prime \prime}}^{1} \in J_{n+1}$. This is ensured by a computation quite similar to the preceding one:

$$
\begin{aligned}
\left|R_{t^{\prime \prime}}^{1}-b_{n+1}\right| & =\left|\Phi^{\prime}\left(R_{t^{\prime \prime}}^{1}\right)-\Phi^{\prime}\left(b_{n+1}\right)\right|=\left|R_{t^{\prime \prime}}^{0}-\Phi^{\prime}\left(b_{n+1}\right)\right| \\
& \leqslant\left|R_{t^{\prime \prime}}^{0}-\Phi^{n+1 \rightarrow n}\left(b_{n+1}\right)\right|+\left|\Phi^{n+1 \rightarrow n}\left(b_{n+1}\right)-\Phi^{\prime}\left(b_{n+1}\right)\right| \\
& <\left|R_{t^{\prime \prime}}^{0}-b_{n}\right|+2 \varepsilon_{n+1},
\end{aligned}
$$

and since (c') gives $\left|R_{t^{\prime \prime}}^{0}-b_{n}\right|<\beta_{n}$, we have $\left|R_{t^{\prime \prime}}^{1}-b_{n+1}\right|<\beta_{n}+2 \varepsilon_{n+1}=\beta_{n+1}$, wherefrom $R_{t^{\prime \prime}}^{1} \in J_{n+1}$.

The last sub-case is when $T<t^{\prime \prime}$. In that case, $0=R_{T}^{1} \in R_{\left[t^{\prime}, T \wedge t^{\prime \prime}\right]}^{1} \subset I_{n+1}$; so we may apply Proposition 7.2 to $t^{\prime \prime}, I_{n+1}, J_{n+1}, R^{1}$ and $T$. We get a sequence $\left(R^{1}, R^{2}, \ldots\right)$ of partial Lévy raises and a r.v. $L \geqslant 1$ such that $R^{L}=R^{1}$ on $[0, T], R_{\left[T, t^{\prime \prime}\right]}^{L} \subset I_{n+1}$ and $R_{t^{\prime \prime}}^{L} \in J_{n+1}$. From $R^{L}=R^{1}$ on $\left[t^{\prime}, T\right]$ and from $(* * *)$, one deduces $R_{\left[t^{\prime}, T\right]}^{L} \subset I_{n+1}$, so $R_{\left[t^{\prime}, t^{\prime \prime}\right]}^{L} \subset I_{n+1}$ and (c") holds. Finally, (a") stems from $R^{N}=R^{1}$ on $\left[0, t^{\prime}\right]$ and from the definition of $R^{1}$ as an $\varepsilon_{n+1}$-double of $Z^{n+1}$.

This terminates the proof of Claim 8.7, and also of Theorem 8.2.

\section{Concluding Remark}

How far is Theorem 8.2 from ergodicity? Can Theorem 8.2 be used in some future proof of ergodicity? Only time will tell; but here is an immediate consequence of topological recurrence.

Call J the $\sigma$-field of measurable, T-invariant subsets of $\mathbb{W}$. Ergodicity amounts to saying that $\mu[G \mid \mathcal{J}]=\mu(G)$ for all open sets $G \subset \mathbb{W}$; Theorem 8.2 only gives:

Corollary 9.1. If $G$ is a non-empty open subset of $\mathbb{W}$, the conditional probability $\mu[G \mid \mathcal{J}]$ is a.e. strictly positive. 
Proof. The J-measurable set $E=\{\mu[G \mid \mathcal{J}]=0\}$ satisfies

$$
\mu(E \cap G)=\int_{E} \mathbf{1}_{G} \mathrm{~d} \mu=\int_{E} \mu[G \mid \mathcal{J}] \mathrm{d} \mu=0 ;
$$

hence, by $\mathbf{T}$-invariance of $E$ and by $\mu$-invariance of $\mathbf{T}$,

$$
\mu\left(E \cap \mathbf{T}^{-n} G\right)=\mu\left(\mathbf{T}^{-n} E \cap \mathbf{T}^{-n} G\right)=\mu\left(\mathbf{T}^{-n}(E \cap G)\right)=\mu(E \cap G)=0 .
$$

Now, the theorem says that $\mu\left(\bigcup_{n \geqslant 0} \mathbf{T}^{-n} G\right)=1$; consequently,

$$
\mu(E)=\mu\left(E \cap \bigcup_{n \geqslant 0} \mathbf{T}^{-n} G\right)=\mu\left(\bigcup_{n \geqslant 0}\left(E \cap \mathbf{T}^{-n} G\right)\right)=0 .
$$

Acknowledgements. I wish to thank Marc Yor and Jean-Paul Thouvenot for their good pieces of advice, and particularly Michel Émery for his great help with the redaction.

\section{REFERENCES}

[1] L.E. Dubins and M. Smorodinsky, The modified, discrete Lévy transformation is Bernoulli, in Séminaire de Probabilités XXVI. Lect. Notes Math. 1526 (1992)

[2] M. Malric, Densité des zéros des transformées de Lévy itérées d'un mouvement brownien. C. R. Acad. Sci. Paris, Sér. I 336 (2003) 499-504.

[3] D. Revuz and M. Yor, Continuous Martingales and Brownian Motion, 3th edition. Springer-Verlag, Berlin (1999) 